\title{
High-resolution $\mathrm{H} \alpha$ imaging of the northern Galactic plane and the IGAPS image database
}

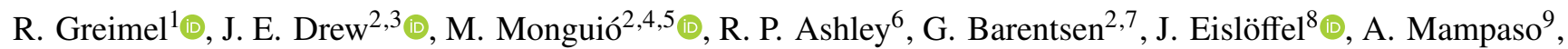

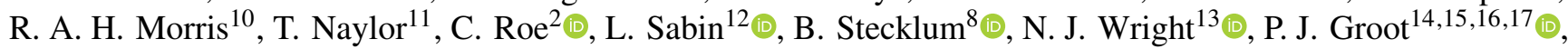

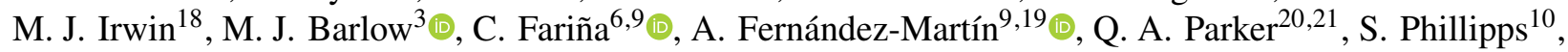 \\ S. Scaringi ${ }^{22}{ }^{(}$, and A. A. Zijlstra ${ }^{23,21}{ }^{(0)}$
}

1 IGAM, Institute of Physics, University of Graz, Universitätsplatz 5/II, 8010 Graz, Austria e-mail: rgreimel@gmail.com

2 School of Physics, Astronomy \& Mathematics, University of Hertfordshire, Hatfield AL10 9AB, UK

3 Department of Physics \& Astronomy, University College London, Gower Street, London WC1E 6BT, UK

${ }^{4}$ Institut d'Estudis Espacials de Catalunya, Universitat de Barcelona (ICC-UB), Martí i Franquès 1, 08028 Barcelona, Spain

5 Universitat Politècnica de Catalunya, Departament de Física, c/Esteve Terrades 5, 08860 Castelldefels, Spain

${ }^{6}$ Isaac Newton Group, Apartado de correos 321, 38700 Santa Cruz de La Palma, Canary Islands, Spain

7 Bay Area Environmental Research Institute, PO Box 25, Moffett Field, CA 94035, USA

8 Thüringer Landessternwarte, Sternwarte 5, 07778 Tautenburg, Germany

9 Instituto de Astrofísica de Canarias, 38205 La Laguna, Tenerife, Spain

10 Astrophysics Group, School of Physics, University of Bristol, Tyndall Av, Bristol BS8 1TL, UK

11 School of Physics, University of Exeter, Exeter EX4 4QL, UK

12 Instituto de Astronomía, Universidad Nacional Autónoma de México (UNAM), AP 106, Ensenada 22800, BC, Mexico

13 Astrophysics Group, Keele University, Keele ST5 5BG, UK

14 Department of Astrophysics/IMAPP, Radboud University, PO Box 9010, 6500 GL Nijmegen, The Netherlands

15 Department of Astronomy, University of Cape Town, Private Bag X3, Rondebosch 7701, South Africa

16 South African Astronomical Observatory, PO Box 9, Observatory 7935, South Africa

17 The Inter-University Institute for Data Intensive Astronomy, University of Cape Town, Private Bag X3, Rondebosch 7701, South Africa

18 Institute of Astronomy, University of Cambridge, Madingley Road, Cambridge CB3 0HA, UK

19 Centro Astronómico Hispano-Alemán, Observatorio de Calar Alto, Sierra de los Filabres s/n, 04550 Gérgal, Almeria, Spain

20 The University of Hong Kong, Department of Physics, Hong Kong SAR, PR China

21 The Laboratory for Space Research, Cyberport 4, Hong Kong SAR, PR China

22 Department of Physics, University of Durham, South Road, Durham DH1 3LE, UK

23 Jodrell Bank Centre for Astrophysics, Alan Turing Building, Manchester M13 9PL, UK

Received 31 March 2021 / Accepted 12 July 2021

\begin{abstract}
The INT Galactic Plane Survey (IGAPS) is the merger of the optical photometric surveys IPHAS and UVEX based on data from the Isaac Newton Telescope (INT) obtained between 2003 and 2018. It captures the entire northern Galactic plane within the Galactic coordinate range $|b|<5^{\circ}$ and $30^{\circ}<\ell<215^{\circ}$. From the beginning, the incorporation of narrow-band $\mathrm{H} \alpha$ imaging has been a unique and distinctive feature of this effort. Alongside a focused discussion of the nature and application of the $\mathrm{H} \alpha$ data, we present the IGAPS world-accessible database of images for all five survey filters, $i, r, g, U_{\mathrm{RGO}}$, and narrow-band $\mathrm{H} \alpha$, observed on a pixel scale of 0.33 arcsec and at an effective (median) angular resolution of 1.1-1.3 arcsec. The background, noise, and sensitivity characteristics of the narrow-band $\mathrm{H} \alpha$ filter images are outlined. Typical noise levels in this band correspond to a surface brightness at full $\sim 1$ arcsec resolution of around $2 \times 10^{-16} \mathrm{erg} \mathrm{cm}^{-2} \mathrm{~s}^{-1} \operatorname{arcsec}^{-2}$. Illustrative applications of the $\mathrm{H} \alpha$ data to planetary nebulae and Herbig-Haro objects are outlined and, as part of a discussion of the mosaicking technique, we present a very large background-subtracted narrowband mosaic of the supernova remnant Simeis 147. Finally, we lay out a method that exploits the database via an automated selection of bright ionised diffuse interstellar emission targets for the coming generation of wide-field massive-multiplex spectrographs. Two examples of the diffuse $\mathrm{H} \alpha$ map output from this selection process are presented and compared with previously published data.
\end{abstract}

Key words. surveys - astronomical databases: miscellaneous - ISM: general - HII regions - planetary nebulae: general ISM: supernova remnants

\section{Introduction}

The stellar and diffuse gaseous content of the Galactic plane continues to be a vitally important object of study as it offers the best available angular resolution for exploring how galactic disc envi- ronments are built and how they operate and evolve over time. For studies of the diffuse interstellar medium (ISM), the optical offers $\mathrm{H} \alpha-$ emission in this strong transition is the pre-eminent tracer of ionised gas. By definition, the ISM is an extended object that must involve investigation by means of imaging data. 
Our purpose here is to present and describe a newly complete resource that enables this style of astronomy, specifically within the gas- and dust-rich northern Galactic plane.

There is a history, stretching back over the last century, of comprehensive surveying of the optical night sky. Until around 1990, much of the wide-area effort depended on photographic emulsions on glass as detectors (e.g. the Palomar, ESO, and UKSchmidt sky surveys, described by Lund \& Dixon 1973; West 1974; Morgan et al. 1992, respectively). The last 30 years have seen a switch to digital detectors that has brought with it the benefits of linearity and increased dynamic range, paving the way for increasingly precise photometric calibration. Thanks to this change and the advance of data science, the community now has access to a number of wide-area broadband surveys offering images at approximately arcsecond angular resolution and point-source catalogues (e.g. SDSS, Pan-STARRS, DECaPS, and Skymapper; see Alam et al. 2015; Chambers et al. 2016; Schlafly et al. 2018; Wolf et al. 2018).

Wide-area narrow-band $\mathrm{H} \alpha$ imaging, our main focus here, has generally been pursued separately from broadband work (most notably VTSS, SHASSA, and WHAM; see Dennison et al. 1998; Gaustad et al. 2001; Haffner et al. 2003). Finkbeiner (2003) merged these surveys into a single map that covers much of the sky, albeit at an angular resolution limited to 6 arcmin. Inevitably, most $\mathrm{H} \alpha$ nebulosity is concentrated in the Galactic plane, along with most of the Galaxy's gas, dust, and stars. Within the plane, the angular resolution needs to be better than this to begin to resolve individual clusters and HII regions that show structure on the sub-arcminute scale. The UK Schmidt H $\alpha$ Survey (SHS; Parker et al. 2005), based on photographic film, has met this challenge in the southern Galactic plane with imaging data of a resolution approaching 1 arcsec. Before the imaging presented here began to be collected, the same could not be said for the plane in the northern hemisphere.

The focus of this paper is full coverage of the plane of the northern Milky Way via digital narrow-band $\mathrm{H} \alpha$ imaging at $\sim 1$ arcsec angular resolution obtained from 2003 up to 2018 using the Wide Field Camera (WFC) on the Isaac Newton Telescope (INT) in La Palma. We showcase the properties of the $\mathrm{H} \alpha$ images and point out different modes of exploitation, past, present, and future. Alongside this we also present the INT Galactic Plane Survey (IGAPS) five-filter image database. IGAPS is the cross-calibrated merger of the two Galactic plane surveys, IPHAS (INT Photometric $\mathrm{H} \alpha$ survey of the northern Galactic plane; Drew et al. 2005) and UVEX (UV-Excess survey of the northern Galactic plane; Groot et al. 2009) ${ }^{1}$. Together, these surveys have offered a new mix of narrow-band $\mathrm{H} \alpha$ alongside four broad bands, spanning the optical. The IPHAS filters were $r / i / \mathrm{H} \alpha$, while the UVEX survey incorporated a repeat in $r$ and $g / U_{\mathrm{RGO}}$ observations. The total IGAPS footprint is a 1850 sq. deg. strip along the Galactic plane defined on the sky in Galactic coordinates $-5^{\circ}<b<+5^{\circ}$ and $30^{\circ}<\ell<215^{\circ}$.

Monguió et al. (2020) have recently presented the merged and calibrated IGAPS point-source catalogue of aperture photometry derived from the IPHAS and UVEX surveys. The focus of this study is on the extraction of stellar photometric data. It did not take on the characterisation of the extended ionised emission traced by the $\mathrm{H} \alpha$ images. This is the partner paper in which this missing piece is put in place.

\footnotetext{
1 In concept, these two surveys are the older siblings to VPHAS+, the survey covering the southern Galactic plane and Bulge (Drew et al. 2014).
}

Here we outline the world-accessible database of IGAPS images that we have set up to hold the $\mathrm{H} \alpha$ (and broadband) data. It is reached via a website that also provides access to the Monguió et al. (2020) point-source catalogues. The imagery we have archived incorporates all IPHAS and UVEX observations, including a minority that did not meet all the desired survey quality criteria. The majority of the included images benefit from the uniform photometric zero points computed by Monguió et al. (2020) when building the IGAPS point-source catalogue.

The structure of the paper is as follows. In Sect. 2 we summarise the relevant features of IGAPS data and our methods as well as the contents of the image database $\mathrm{e}^{2}$. This is followed by an outline of the search tool available for querying the database (Sect. 3). Attention then switches to our main focus: the properties and application of the narrow-band $\mathrm{H} \alpha+[\mathrm{NII}]$ imagery. Section 4 sets the ball rolling with an overview of the sensitivity and the nature of the background captured in the narrow-band filter. We then go on, in Sect. 5, to highlight two contrasting examples of its exploitation for the science of diffuse nebulae (planetary nebulae and Herbig-Haro objects). A discussion of techniques for mosaicking the $\mathrm{H} \alpha$ data then follows (Sect. 6). Looking to the near future of massively multiplexed optical spectrographs, we present a method for automated searching of the IGAPS image database for high $\mathrm{H} \alpha$ surface brightness nonstellar diffuse-ISM spectroscopic targets (Sect. 7). To round off, we show two contrasting examples of the output from the automated search in Sect. 7.4.

\section{Description of the image database}

A series of papers (Drew et al. 2005; González-Solares et al. 2008; Groot et al. 2009; Barentsen et al. 2014; Monguió et al. 2020) has already described the survey data acquisition and pipelining. So here it is only necessary to repeat pertinent details.

The camera used, the INT's WFC, is a mosaic of four chargecoupled devices (CCDs) arranged in an L shape with a pixel size of 0.33 arcsec pixel $^{-1}$. Each CCD images a sky region of roughly $11 \times 22$ sq. arcmin, giving a total combined field per exposure of approximately $0.22 \mathrm{sq}$. deg. The five filters used $-U_{\mathrm{RGO}}, g, r$, $i$, and a narrow-band $\mathrm{H} \alpha$ - have central wavelengths of 364.0, 484.6, 624.0, 774.3, and 656.8 nm, respectively. Despite the filter's different naming, the $U_{\mathrm{RGO}}$ transmission curve quite closely resembles that of Sloan $u$ (Doi et al. 2010). We shall refer to the narrow-band filter as the $\mathrm{H} \alpha$ filter throughout this work, but we note for completeness that the $95 \AA$ bandpass also captures the [NII] 654.8, 658.4 nm forbidden lines typical of HII-region emission. The numbers of CCD images per filter in the repository are listed along with other performance parameters in Table 1. UVEX and IPHAS $r$ band observations are distinguished by labelling them $r_{U}$ and $r_{I}$, respectively.

IPHAS and UVEX shared the same footprint and set of pointings, such that the northern Galactic plane was covered via 7635 WFC pointings, tessellating the survey area with, typically, a small overlap. In addition, each field was observed again, and usually around 5 min later, at an offset of +5 arcmin in RA and +5 arcmin in Dec in order to fill in the gaps between the CCDs and also to minimise the effects of bad pixels and cosmic rays. As a result, almost all locations in the northern plane have been imaged twice in either survey. The presence of the $r$ band in both surveys means that there will usually be at least 4 CCD images,

\footnotetext{
2 Available at http://www . igapsimages.org/
} 
Table 1. Properties of the repository contents by filter for the better-quality image sets graded A to C.

\begin{tabular}{|c|c|c|c|c|c|c|c|c|}
\hline Filter & ING/WFC name & $N$ & $\begin{array}{l}\text { Exposure } \\
\text { (s) }\end{array}$ & $\begin{array}{c}\text { PSF } F W H M \\
\quad(\operatorname{arcsec})\end{array}$ & Sky count & $\begin{array}{c}\mathrm{ZP} \\
\text { (mag.) }\end{array}$ & $\begin{array}{l}5 \sigma \text { depth } \\
\text { (mag.) }\end{array}$ & Moon phase \\
\hline$i$ & WFCSloanI & 83652 & $10(83 \%)$, and $20(17 \%)$ & 1.06 & 92 & 26.42 & 20.28 & 0.68 \\
\hline $\mathrm{H} \alpha$ & WFCH6568 & 83652 & 120 & 1.20 & 57 & 26.57 & 20.40 & $" \prime$ \\
\hline$r_{I}$ & WFCSloanR & 83652 & $30(85 \%)$, and $10(15 \%)$ & 1.16 & 164 & 28.19 & 21.37 & " \\
\hline$r_{U}$ & " & 67896 & 30 & 1.18 & 126 & 28.20 & 21.67 & 0.21 \\
\hline$g$ & WFCSloanG & 67895 & 30 & 1.26 & 61 & 28.71 & 22.38 & $\prime \prime$ \\
\hline$U_{\mathrm{RGO}}$ & WFCRGOU & 67892 & 120 & 1.48 & 43 & 27.85 & 21.47 & " \\
\hline
\end{tabular}

Notes. The number $N$ specifies the number of CCD images available in the A to C grade range for each filter (D-grade images with identified problems are not included in the count). Median values are listed for the full width half maximum of the point spread function (PSF FWHM), the sky background count, zero point (ZP), $5 \sigma$ detection limit in the Vega system, and moon phase. The PSF FWHM and the background count are pipeline measures, while the ZP and limiting magnitude are based on the uniform calibration (Monguió et al. 2020). Moon phase is given as a fraction, such that 0 corresponds to new moon and 1 to full moon. The $r_{I}$ exposure time started out at $10 \mathrm{~s}$ but was raised to $30 \mathrm{~s}$ from 2004 on, while the $i$ exposure time was increased to $20 \mathrm{~s}$ starting in October 2010.

uniquely in this band, covering any given position within the footprint. The only exception to mention is that there is a triangular patch of sky towards $\ell \sim 215^{\circ}$ where there are no UVEX $U_{\mathrm{RGO}}, g, r_{U}$ images.

A distinction between the two surveys is that the blue UVEX data were obtained during dark time, while IPHAS observations were generally made with the moon above the horizon. This difference means that background sky counts are typically higher in the red IPHAS images than they are in UVEX images. A practical consequence of this is that UVEX $r_{U}$ data go deeper than IPHAS $r_{I}$ by $0.3 \mathrm{mag}$, on average (see Table 1). Another impact is that the background levels in the brighter-time observations in the $\mathrm{H} \alpha$ filter can exhibit marked variation, depending on how far away and full the moon is and on cloud cover (see Sect. 4). These variations, especially when mixed with $\mathrm{H} \alpha$ nebulosity, represent a challenge when mosaicking IPHAS data to build large-area images. How this challenge can and has been met is the subject of Sect. 6 .

A part of the pipelining procedure for all survey data was to apply a flux calibration based on nightly standard-star observations. The zero points, in the Vega system, obtained on this basis remain associated with all images in the repository and are stored in every image header as the keyword MAGZPT. The meaning of MAGZPT is that it is the magnitude in the Vega system of an object giving 1 count per second (it does not fold in the exposure time; for details on how it is derived, see Eq. (4) in González-Solares et al. 2008). Experience has shown that broadly consistent and reliable results were obtained for the $U_{\mathrm{RGO}}$ and $\mathrm{H} \alpha$ filters if their zero points were fixed at a constant offset relative to (respectively) their partner $g$ and $r_{I}$ frames. For $U_{\mathrm{RGO}}$ this is the only calibration presently available. For $g, r_{U}, r_{I}$, $i$ and $\mathrm{H} \alpha$, a uniform recalibration of the photometry was undertaken in preparing the point-source catalogue (Monguió et al. 2020). The result of this is that around $2 / 3$ of the images in the repository now carry a revised zero point (PHOTZP header keyword) that rests on a comparison with Pan-STARRS $g, r$ and $i$ photometry. This zero point has incorporated the image exposure time, which means it is the magnitude of a source giving 1 count integrated over the exposure. Hence, the Vega magnitude of any imaged source can be computed from:

$m=-2.5 \log$ (enclosed counts $)+$ PHOTZP,

where, for a point source, the enclosed counts would be the total counts within a user-specified aperture, after subtraction of an estimate for the enclosed underlying background.
The $\mathrm{H} \alpha$ filter magnitude scale is not one conventionally defined within the Vega system. This means we need to define the flux corresponding to the zero of the magnitude scale appropriate to it. We determine that the zero-magnitude flux entering the top of the Earth's atmosphere within the WFC H $\alpha$ filter transmission profile is

$F[m(\mathrm{H} \alpha)=0]=1.57 \times 10^{-7} \mathrm{ergs} \mathrm{cm}^{-2} \mathrm{~s}^{-1}$.

In the $\mathrm{AB}$ magnitude system this is equivalent to a magnitude of 0.328 .

Expressed in point-source magnitudes the bright limit of the images is in the region of $11-12$ in $i$ and $\mathrm{H} \alpha$, rising to $12-13$ in the more sensitive $r$ and $g$ bands. There were specific observation periods in which WFC electronic issues meant that saturation was reached at appreciably lower count levels than the norm. For example, there was a particular problem affecting CCD 2 in November 2006 that would push the bright limit fainter by up to a magnitude. Users of the data can track such problems in the images via the count level recorded against the header keyword SATURATION.

As part of creating the point-source catalogue images were graded from best to worst as $\mathrm{A}++, \mathrm{A}+$, and $\mathrm{A}$ to $\mathrm{D}$. A definition of the grades is available in Appendix A of Monguió et al. (2020). A last point to make about the images is to note that while there is minimal vignetting of CCDs 1,2 , and 4 , there is persistent vignetting of the corner of CCD 3 farthest from the optical axis of the WFC (which passes through CCD 4). In Appendix A.2 a typical confidence map is shown to illustrate this. In Appendix A.3 we briefly outline common artefacts in the image data.

\section{Accessing the images}

All the reduced images from both surveys can be accessed via the website ${ }^{3}$ hosted by University College London. Altogether the repository contains 527736 CCD frames, of which 314923 (or $60 \%$ ) carry the best-quality A grades and 73097 (14\%) are minimum-quality D graded. These grade assignments were made at the level of the basic unit of observation in the two constituent surveys - the consecutive trio of exposures obtained at each sky position. The details of the grading system at work in evaluating data for the IGAPS catalogue can be found in

\footnotetext{
3 http://WWW.igapsimages.org/
} 
Appendix A of the catalogue paper (Monguió et al. 2020). A consequence of this approach, also applied to the earlier IPHAS DR2 release (Barentsen et al. 2014), is that the grade assignment, referred to individual CCD frames or even filters, can be pessimistic, as it takes just one substandard exposure in a set of three to pull down the grade for all of them. In view of this, and the occasional scientific value of maximising the number of images to examine, the decision was taken to retain all $\mathrm{D}$ graded data in the repository, alongside the $\mathrm{A}$ to $\mathrm{C}$ graded exposures.

The images access page within the website offers a search tool that enables users to search for and download images that either overlap a single specified position or occupy a square box of size up to $1 \times 1 \mathrm{sq}$. deg. on the sky. The user can choose the filters of interest and decide whether to omit grade D data. In response to a query, the tool returns a table listing the images meeting requirement, along with key metadata (grade, seeing, depth, whether calibrated) that can inform the user's final choice of images for download. For convenience, there is a column of tick boxes in the table that allows the user to deselect some of the listed images before initiating the download of a gzipped or tarred collection of Rice-compressed (.fz) images.

It is generally the case that a contemporaneous $r_{I}$ image accompanied an $\mathrm{H} \alpha$ and $i$ image of the same pointing. Similarly the UVEX $r_{U}, g$ and $U_{\mathrm{RGO}}$ images were observed as consecutive triplets. Given that stars are sometimes subject to variability, users of the repository may need to bear this in mind when deciding how to select images for scientific exploitation.

The website also provides a link to a large table of metadata that previews the header information provided with the full set of image profiles.

\section{Properties of the $\mathrm{H} \alpha$ images: Backgrounds and sensitivity}

At the outset, the IPHAS survey was allocated time on the expectation that the programme could cope with a moonlit sky. After the first few seasons and some experience had accumulated, the brightest nights were increasingly avoided. Indeed, in the late stages when the acquisition of the blue UVEX filters took priority, dark and grey nights became the norm. The net result is that the median background level among all the uniformly calibrated $\mathrm{H} \alpha$ images is closer to grey, than bright. Table 2 provides some numerical detail illustrating the strongly skewed distribution finally achieved. For comparison with the magnitudes in the table, we mention that the Isaac Newton Group (ING) exposure time calculator uses $20.6,19.7$ and $18.3 \mathrm{mag} \operatorname{arcsec}^{-2}$ to represent dark, grey and bright sky in the $R$ photometric band - values closely resembling the tabulated 5 th, 50 th, and 95 th percentiles.

Around a third of the images were not passed through uniform calibration: of these (generally inferior) data, just under a third have background levels in $\mathrm{H} \alpha$ exceeding the 210 counts marking the 95th percentile of the uniformly calibrated set of images. Most of them were obtained early on in the survey, and most have been repeated, resulting in calibrated alternatives. Just 339 calibrated $\mathrm{H} \alpha$ exposures (1356 CCD frames, or $2 \%$ of the total) are left without alternatives in the database, where the background count exceeds the 95th percentile of 210 counts. Where these sit in the survey footprint is shown in Fig. 1.

Figure 2 shows how the recorded sky background count levels convert into narrow-band $\mathrm{H} \alpha$ surface brightness, via the set of zero points, PHOTZP. The plot includes only data that have passed through the uniform photometric calibration. Most of the data conform reasonably well to a linear trend such that 1 count per pixel corresponds to $3.32 \times 10^{-17} \mathrm{ergs} \mathrm{cm}^{-2} \mathrm{~s}^{-1} \operatorname{arcsec}^{-2}$
Table 2. Percentiles of the distribution of $\mathrm{H} \alpha+[\mathrm{NII}]$ background levels.

\begin{tabular}{lccc}
\hline \hline \multirow{2}{*}{ Quantity } & \multicolumn{3}{c}{ Percentiles } \\
\cline { 2 - 4 } & 5 & 50 & 95 \\
\hline Counts & 27 & 52 & 210 \\
$\begin{array}{l}\text { Vega magnitude } \\
(\text { mag arcsec }\end{array}$ & & & \\
$\begin{array}{l}\text { Surface brightness } \\
\left(10^{-15} \mathrm{erg} \mathrm{cm}^{-2} \mathrm{~s}^{-1} \operatorname{arcsec}^{-2}\right)\end{array}$ & 0.91 & 1.7 & 7.4 \\
\hline
\end{tabular}

Notes. These are pipeline-computed values obtained for uniformly calibrated images. The sample used here numbers 63956 CCD frames (or 15989 WFC exposures). Not included are the 36808 CCD frames (9202 WFC exposures) for which only the pipeline photometric calibration is available.

(or 5.9 rayleighs) ${ }^{4}$. The absolute minimum sky brightness measured in any calibrated frame is $6.6 \times 10^{-16} \mathrm{ergs} \mathrm{cm}^{-2} \mathrm{~s}^{-1} \operatorname{arcsec}^{-2}$ ( $\sim 120$ rayleighs), but it is commonly more than twice this. Sky transparency necessarily influences the behaviour. At times of reduced transparency the sensitivity suffers, driving data points from affected nights onto steeper linear trends in Fig. 2.

Figure 3 is a plot of the pipeline measurement of sky noise versus the estimate of sky background, both in counts per pixel. For around a half of the uniformly calibrated frames, the noise is limited to under 6 counts per pixel (or $\sim 2 \times$ $10^{-16} \mathrm{ergs} \mathrm{cm}^{-2} \mathrm{~s}^{-1} \operatorname{arcsec}^{-2}$ ), and it is extremely rare that the sky noise is more than $\sim 12$ counts per pixel. A simple expectation for the form of the relation between sky noise and level is

$N_{\mathrm{bg}}=\left(N_{\mathrm{RN}}+\sqrt{C_{\mathrm{bg}}}\right) / \sqrt{n_{\mathrm{pe}}}$,

where $N_{\mathrm{bg}}$ and $C_{\mathrm{bg}}$ are respectively the sky noise and sky level, $N_{\mathrm{RN}}$ is the CCD read noise, also in counts per pixel, and $n_{\mathrm{pe}}$ represents the effective number of pixels over which the sky statistics are measured. Basically, we expect the noise to be the sum of a constant and a Poisson component. In practice, the sky level is determined numerically by the pipeline using two-dimensional non-linear background tracking across the full CCD at a superpixel level. After the background fit has been subtracted from an image, the sky noise is calculated iteratively from the clipped median absolute deviation (MAD) of the residuals.

As the read noise for the WFC is known to be $N_{\mathrm{RN}}=2.37$ counts, we only need to fit one parameter of this function. Before the fitting, outliers above the dashed line in Fig. 3 were removed. The fit was performed using an iterative 3- $\sigma$ clipped LevenbergMarquardt algorithm using the median and MAD in place of mean and $\sigma$. The result of the fit is

$N_{\mathrm{bg}}=\left(2.37+\sqrt{C_{\mathrm{bg}}}\right) / \sqrt{2.88}=1.4+0.589 \sqrt{C_{\mathrm{bg}}}$.

Allowing an additional fit parameter, namely a multiplicative factor for the $\sqrt{C_{\mathrm{bg}}}$ term, does not lead to a statistically significant improvement in the fit, and the factor is found to be very close to 1 . Hence we are confident that Eq. (3) accurately describes the distribution.

In presenting the SHS, Parker et al. (2005) compared its sensitivity with IPHAS and other available $\mathrm{H} \alpha$ wide-area surveys. In their Table 1 it was estimated that the depth reached by the narrow-band (IPHAS) data presented here is $\sim 3$ rayleighs. This is a surface brightness of $1.7 \times 10^{-17} \mathrm{ergs} \mathrm{cm}^{-2} \mathrm{~s}^{-1} \operatorname{arcsec}^{-2}$ or, as required by the mean calibration illustrated in Fig. 2, the

4 At $\mathrm{H} \alpha, 1$ rayleigh is equivalent to $5.67 \times 10^{-18} \mathrm{ergs} \mathrm{cm}^{-2} \mathrm{~s}^{-1} \operatorname{arcsec}^{-2}$. 


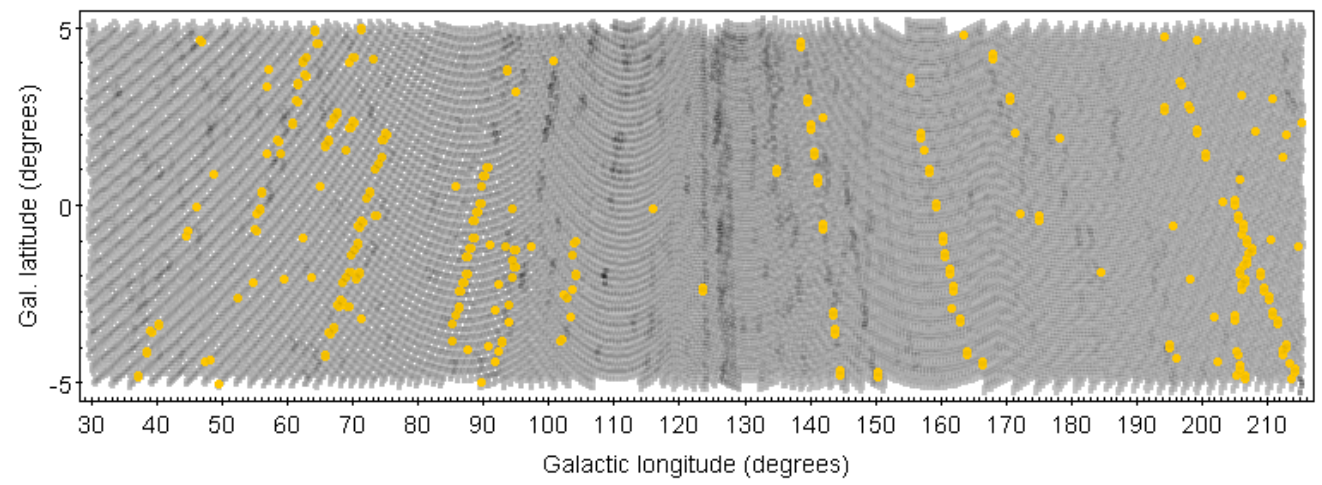

Fig. 1. Map of the uniformly calibrated IGAPS field centres (grey). A darker grey colour is seen where the database contains more than one uniformly calibrated exposure. The orange overplotted points mark the 339 exposure sets (1356 CCD frames) without alternatives in the database, where the $\mathrm{H} \alpha$ sky background exceeds 210 counts ( $>95$ th percentile).

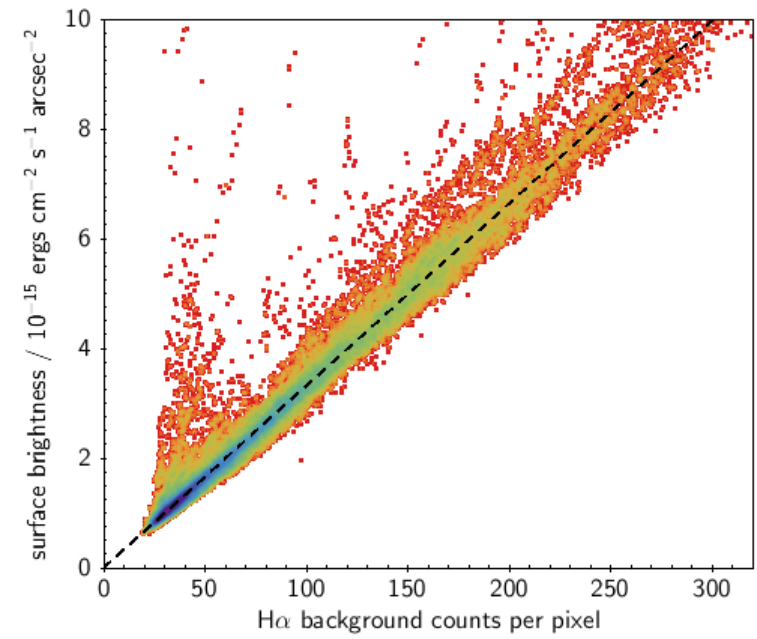

Fig. 2. Density plot of background surface brightness in the $\mathrm{H} \alpha$ band as a function of measured mean sky level in counts. The colour scale is logarithmic, with blue representing the highest density of points. The images used to build this diagram are the IGAPS uniformly calibrated set, which enables validated conversion of the sky counts to surface brightness via each image's zero point. The black dashed line has a gradient of $3.32 \times 10^{-17} \mathrm{ergs} \mathrm{cm}^{-2} \mathrm{~s}^{-1} \operatorname{arcsec}^{-2}$ per count.

equivalent of around a half count per pixel in a 120 s exposure at the typical 1 to $1.5 \mathrm{arcsec}$ seeing. The on-sky solid angle that the Parker et al. (2005) estimate refers to was not made explicit - clearly it cannot be $1 \operatorname{arcsec}^{2}$. If we assume $C_{\text {bg }}=0.5$ counts pixel ${ }^{-1}$ at $3 \sigma, N_{\text {bg }}=0.5 / 3$, we can use Eq. (3) to calculate the pixel area needed to achieve a sensitivity of 3 rayleighs. We obtain $18.5 \times 18.5$ pixels or $6.1 \times 6.1$ arcsec.

Used at full seeing-limited resolution, the $\mathrm{H} \alpha$ data provide safe detection of raised surface brightness due to diffuse-ISM emission at the level of a few times $10^{-16} \mathrm{ergs} \mathrm{cm}^{-2} \mathrm{~s}^{-1} \operatorname{arcsec}^{-2}$, or 50-100 rayleighs. That this is so will be demonstrated by different means in Sect. 7.

\section{Exploitation of the $\mathrm{H} \alpha$ images}

The search for and characterisation of extended nebulae was an original goal of the IPHAS component of the merged IGAPS survey. Initially this mostly concentrated on planetary nebulae (PNe). Discoveries of examples of those alternative products of end-state stellar evolution - supernova remnants (SNRs) - have also been found and recorded (Sabin et al. 2013).

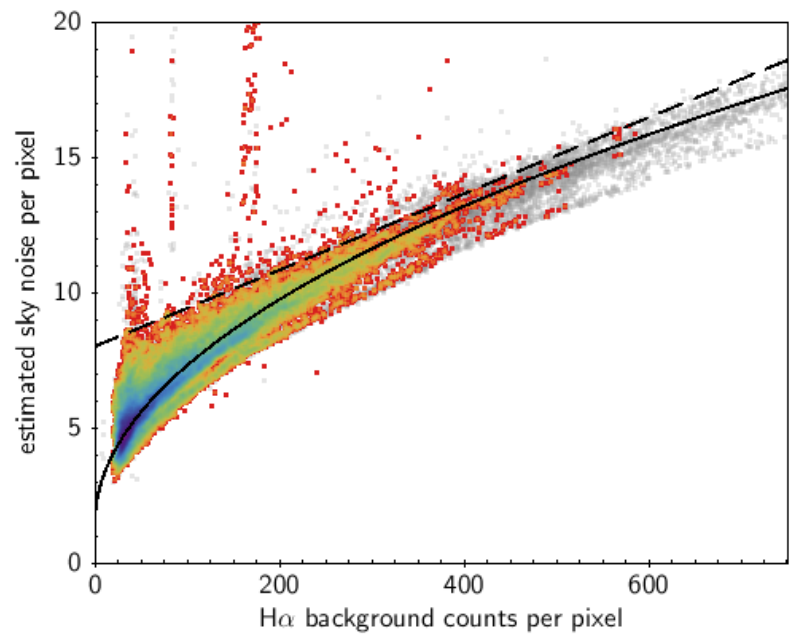

Fig. 3. Density plot of the pipeline-measured sky noise in the $\mathrm{H} \alpha$ filter as a function of sky level. Both are in units of counts per pixel. The coloured data shown, and plotted on a logarithmic density scale, are drawn from the IGAPS uniformly calibrated set. The solid black line is the fit discussed in the text, given as Eq. (4), that shows that the sky noise increases mainly as the square root of the sky level. The lighter grey data (underneath) are from images with zero points that were not passed through uniform photometric calibration.

Below we present brief discussions of how nebulae can be found and studied, taking the contrasting examples of new PNe and - from the more obscured first phase of the stellar life cycle - a Herbig-Haro object (HHO).

\subsection{Planetary nebulae}

Planetary nebulae are the end-products of low and intermediate mass stars $\left(\sim 1-8 M_{\odot}\right)$ where a hot central star fully ionises its surrounding shell leading to a glowing nebula. These objects are ideal tools to study the later stages of stellar evolution as most stars in our Galaxy will go through this phase. We can access information related to their physical characteristics via plasma diagnostics (density, temperature, velocity, and so on) and the chemical composition allows the measurement of their impact on the chemical enrichment of any given galaxy. However, most of the known PNe are bright and/or nearby as the faint ones have been ignored or are out of reach due to observational constraints. An aim of IPHAS was to perform a near complete 
census of the PNe in the northern Galactic plane, where a higher concentration of ionised sources co-exists with high extinction. Progress towards this goal has been described by Sabin (2008) and Sabin et al. (2014).

\subsubsection{Detection methods}

Depending on the size of the sources two methods were adopted. On the one hand, compact or point-source PNe have been selected based on $\mathrm{H} \alpha$ excess as measured and recorded in photometric catalogues, and cross-checked against IR photometry (e.g. 2MASS; see Viironen et al. 2009). On the other hand, for the case of extended $\mathrm{PNe}$, a mosaicking process was developed using $2^{\circ} \times 2^{\circ} \mathrm{H} \alpha-r$ mosaics with $5 \times 5$ pixels and $15 \times 15$ pixels binning factors (corresponding to $\sim 1.7$ and $\sim 5 \operatorname{arcsec}$ effective pixel sizes, respectively; see Sabin 2008). The coarser binning was mainly used to detect large and low-surface-brightness nebulae, while the lower binning was aimed at detecting smaller nebulae hidden in crowded stellar fields. In the latter case we also used the technique adopted in the southern MASH survey (Parker et al. 2006; Miszalski et al. 2008) where composite imagery ( $\mathrm{H} \alpha, r^{\prime}$, and $i^{\prime}$ filters) is used to distinguish stars from diffuse ionised nebulae ${ }^{5}$. Always, new objects were confirmed by independent eyeballing of the data by several team members.

This task was found to be ideal for undergraduate projects and two example discoveries are shown below.

\subsubsection{New discoveries}

Hundreds of new PN candidates were found as a result of the visual search with the mosaics and a first large spectroscopic follow-up involving no less than nine worldwide telescope and instrument pairings. ranging from $1.5 \mathrm{~m}$ (ALBIREO spectrograph at the Observatory of Sierra Nevada, Spain) to $10.4 \mathrm{~m}$ (GTC-OSIRIS spectrograph at the Observatorio del Roque de los Muchachos, Spain) was conducted (Sabin et al. 2014). We identified 159 objects as True (113), Likely (26) and Possible (20) PNe and unveiled a large range of shapes (mostly elliptical, bipolar and round $\mathrm{PNe}$ ) and sizes (up to $\sim 8 \mathrm{arcmin}$ ).

Among all the newly detected $\mathrm{PNe}$, some caught our attention first due to their outstanding morphology and then due to their interesting characteristics revealed by subsequent deep analyses. Notable examples include: the knotty bipolar IPHASX J194359.5+170901, now known as the Necklace Nebula (Corradi et al. 2011); the quadrupolar IPHASX J012507.9+635652 (alternately named Príncipes de Asturias by Mampaso et al. 2006); IPHASX J052531.19+281945.1 at large galactocentric distance (Viironen et al. 2011); and finally, the PN discovered around the nova V458 Vul (Wesson et al. 2008). IPHAS, and now IGAPS, also allows us to target the particular group of PNe interacting with the ISM. Those objects that are slowly diluting in the ISM are particularly difficult to detect most of all in their late phase. In this case IPHAS imaging data can reveal such faint material (Sabin et al. 2010).

We show in Figs. 4 and 5 two of the many new PNe that have been found and have not yet appeared in the refereed literature. Most of the new finds are published in Sabin et al. (2014). The first object IPHASX J015624.9+652830 (PNG 129.6+03.4), shown in Fig. 4, came from parallel searches of the $15 \times 15$ pixel binned $\mathrm{H} \alpha-r$ (5 arcsec per pixel) mosaics, carried out by team

\footnotetext{
5 With advances in the use of machine learning, the classifications of objects in the HASH database can now be automated (Awang Iskandar et al. 2020).
}

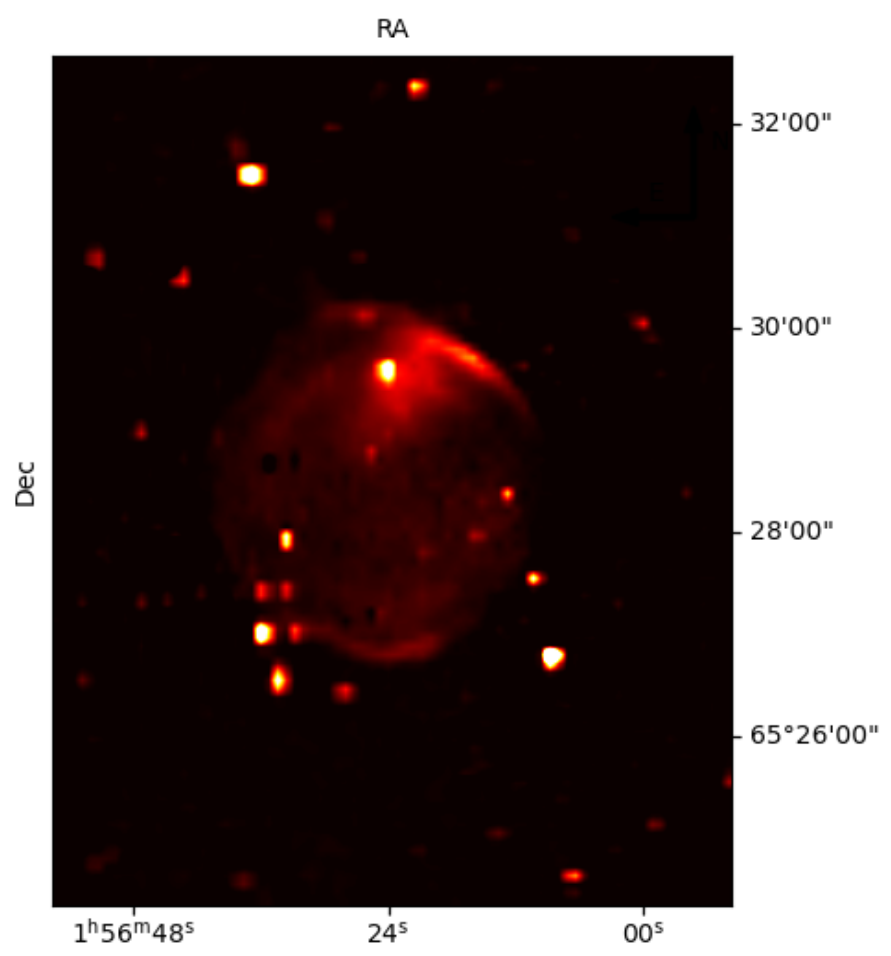

Fig. 4. IPHASX J015624.9+652830. This is a cutout from one of the 2 by 2 degree binned $\mathrm{H} \alpha-r$ mosaics used for searching for new PNe.

RA

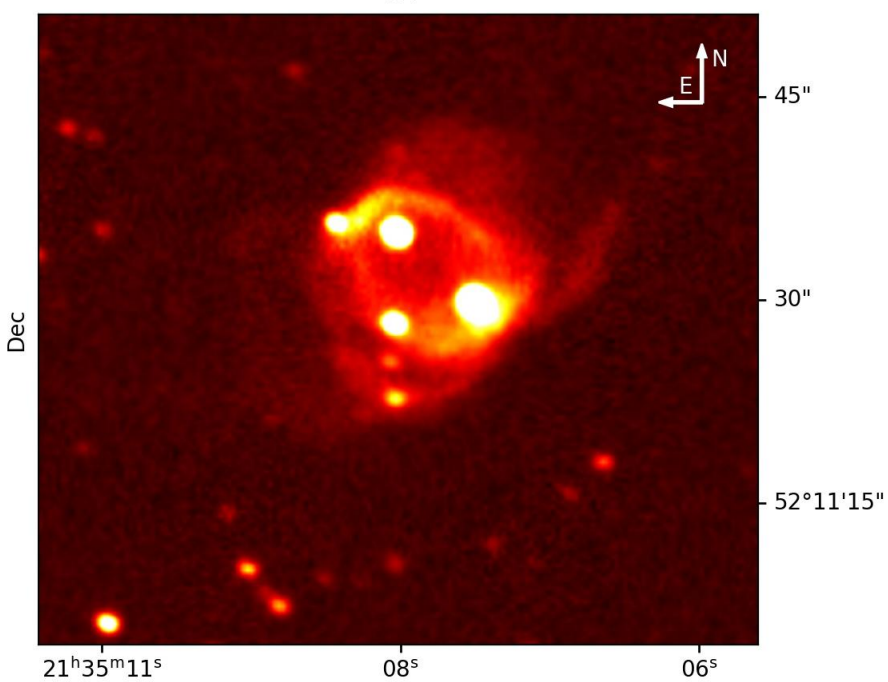

Fig. 5. PNG 95.3+0.2. This is a cutout from one of two CCD frames downloaded from the IGAPS image archive. The alternative available image of the PN (not shown) happens to sit adjacent to a 'bad line' in the CCD. It is precisely to mitigate against this kind of problem that the survey strategy was to collect data from paired offset pointings. The object IPHAS J213508.15+521128.0, discussed in the text, is the lower left of the three bright stars in the central region of the nebula.

members and undergraduate students supervised by them in 2007. This object can be seen in the $\mathrm{H} \alpha$ images but is easily missed, yet it stands out very clearly in the binned difference images as the only object in an otherwise blank frame. This demonstrates the usefulness of this method for the discovery of new objects, especially those that, like this one, are only detectable in optical wavebands. 
The second nebula, PNG95.3+0.2, shown in Fig. 5, was discovered using the same search method by Fernández-Martín (2007). Subsequent high-resolution (0.6 arcsec seeing) imaging with the NOT telescope on the night of 4 September 2007, through $\mathrm{H} \alpha$, [OIII] $5007 \AA$, and [NII] $6583 \AA$ filters further clarified an intricate morphology with a bright, roughly elliptical central shell, and a pair of fainter twisted protrusions that show up especially well in the [NII]-only image. Since the IPHAS $\mathrm{H} \alpha$ filter bandwidth also incorporates this line, Fig. 5 is a composite image, showing both the [NII]-dominated outer filamentary structure and the $\mathrm{H} \alpha$ dominated inner ellipse.

PNG 95.3+0.2 is now listed in the HASH Catalogue of Parker et al. (2016). It is associated with an infrared (WISE, and $90 \mu \mathrm{m} \mathrm{AKARI}$ ), and $1420 \mathrm{MHz}$ radio source (Taylor et al. 2017). According to Anders et al. (2019), the $G \simeq 17.1 \mathrm{mag}$ star near the geometrical centre, IPHAS J213508.15+521128.0, or Gaia EDR3 2171830374492778880, is a distant, reddened, and apparently relatively cold $\operatorname{star}\left(D=5.5 \pm 0.9 \mathrm{kpc}, A_{V}=\right.$ $\left.3.9 \pm 0.2 \mathrm{mag}, T_{\text {eff }}=4800 \pm 260 \mathrm{~K}\right)$. However, our evaluation of the IGAPS broadband photometry (Monguió et al. 2020) is that the available magnitudes are also consistent with this object being a much hotter, even more extinguished star. The other two, brighter stars embedded in the nebula are located in the foreground at much more secure parallax-based distances of 0.9 and $1.6 \mathrm{kpc}$ : In the Anders et al. (2019) database they too are assigned low $T_{\text {eff }}$ values, incompatible with those of a hot PN central star. Vioque et al. (2020) combine IPHAS, 2MASS, and WISE data in a search for new Herbig Ae/Be stars and list IPHAS J213508.15+521128.0 as a non-Herbig AeBe, non-premain-sequence, and non-classical Be star - they also do not confirm an association with a PN (their PN flag is empty). The WISE source, detected in the four bands and centred at 1.1 arcsec from IPHAS J213508.15+521128.0, shows red IR colours like known PNe, while the spectral energy distribution of the star, built from Pan-STARRS, 2MASS, ALLWISE, and AKARI data, is typical of a reddened star up to the WISE $W 3(12 \mu \mathrm{m})$ band. Beyond that, a strong IR excess appears up to $90 \mu \mathrm{m}$, and points to a physical association of IPHAS J213508.15+521128.0 with the nebula. If that is the case, the apparent nebular size of around 30 arcsec would imply a rather large, evolved nebula, $0.8 \mathrm{pc}$ in diameter, and also suggest the existence of a hidden hot star (possibly a binary) in the surroundings. PNG 95.3+0.2 is an appealing example of an IPHAS extended object with plenty of online, publicly available information; it nevertheless deserves further dedicated observations, including careful quantitative spectroscopy to pin down the central star.

Basic confirmation spectra exist for both the above nebulae. The objects have also been independently discovered more recently by amateur astronomers. The first object, IPHASX J015624.9+652830, has also come to be known as Ferrero 6, Fe6, and PNG129.6+03.4, while the second, PNG 95.3+0.2, is also known as StDr Objet 1. Full details on both can be found in the HASH database (Parker et al. 2016).

\subsubsection{Previously known $\mathrm{PNe}$}

The survey is also useful for the re-analysis of already known PNe. IPHAS images can unveil new faint structures associated with known PNe that have hitherto evaded detection. A clear example of this is the detection of the extended tail of the known Sh 2-188 by Wareing et al. (2006), which enabled the reevaluation of its full extent. A different application has recently been presented by Dharmawardena et al. (2021): with a view to appraising different methods of determining PN distances, they

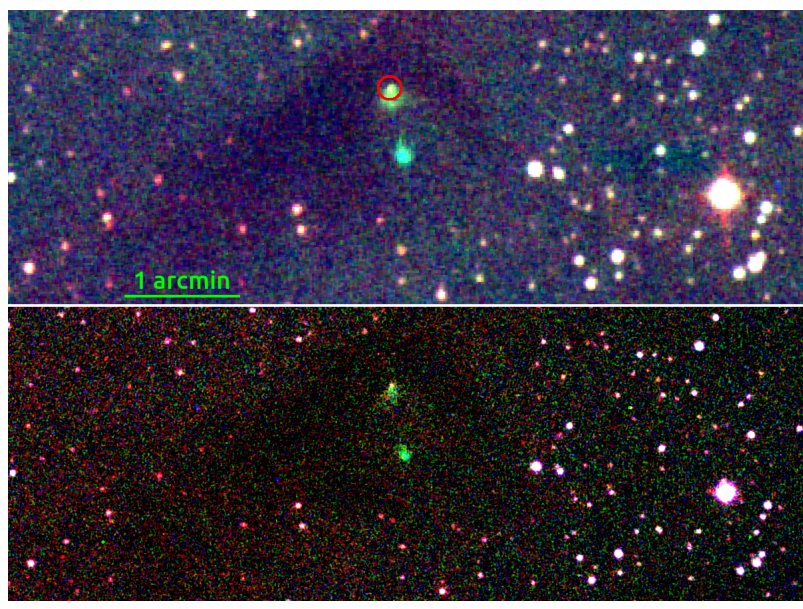

Fig. 6. Comparison of composite images of the dark cloud Dobashi 3782 and a contained HHO. Top: composite image (epoch 2007) based on $I, \mathrm{H} \alpha$, and [SII] frames taken with the Tautenburg Schmidt telescope. It hosts the YSO IRAS 01166+6635, marked by the circle, which drives a jet that excites a compact HHO south of it (centre). It appears greenish-blue in this representation because of its strong $\mathrm{H} \alpha$ emission. Bottom: IGAPS composite image (epoch 2013), based on $i, \mathrm{H} \alpha$, and $r_{I}$ frames. The YSO, as well as the HHO, appears green because it is not clearly detected in either $r_{I}$ or $i$. The HHO is just resolved at no more than 2 arcsec across.

have collected IPHAS H $\alpha+[\mathrm{N}$ II $]$ aperture photometry fluxes for 151 previously known nebulae as well as for 46 confirmed or possible PNe that had been discovered by the IPHAS survey.

\subsection{Herbig-Haro objects}

During their growth, young stellar objects (YSOs) eject a fraction of the in-falling matter at high speed via bipolar jets and outflows. Their shock fronts, delineated by line emission, particularly in $\mathrm{H} \alpha$, [SII], and [OI], are called Herbig-Haro objects (HHOs; Herbig 1950; Haro 1952). These objects not only trace the presence of young stars but can also serve as a record of the accretion history. The kinematics of HHOs, derived from proper motions (PMs) and radial velocities (RVs), allow a kinematic dating of the ejection event. Moreover, such data provide information on the inclination $i$ of the circumstellar accretion disc. Constraining the latter is crucial for the analysis of YSO spectral energy distributions using radiative transfer modelling.

The potential of IGAPS for such studies is illustrated by the example of a hitherto unknown HHO, driven by IRAS 01166+6635. This low-mass YSO (Connelley et al. 2008) is emerging from the small dark cloud, Dobashi 3782, situated at a kinematic distance of $240 \mathrm{pc}$ (Wouterloot \& Brand 1989). Narrow-band imaging performed in 2007 with the Tautenburg Schmidt telescope revealed a compact HHO south of the YSO within $\sim 1$ arcsec of the position RA 01:20:02.9, Dec +66:51:00 (J2000) (Fig. 6). The estimated extinction out to the distance of this cloud is about $A_{V} \sim 2.5$, averaged across a few arcminutes (Sale et al. 2014). The extinction towards the optically faint YSO is without doubt much more. At the position of this YSO there are eight entries in the IGAPS catalogue (Monguió et al. 2020) within a radius of 5 arcsec. Six of the eight are $\mathrm{H} \alpha$ only sources.

Blinking with the POSS1 red image showed evidence for PM within $\sim 50$ years. Thus, in order to establish its kinematics, $\mathrm{H} \alpha$ and $R$-band frames have been secured for as many epochs as possible. Four $\mathrm{H} \alpha$ frames with grade A quality (r367494 and 


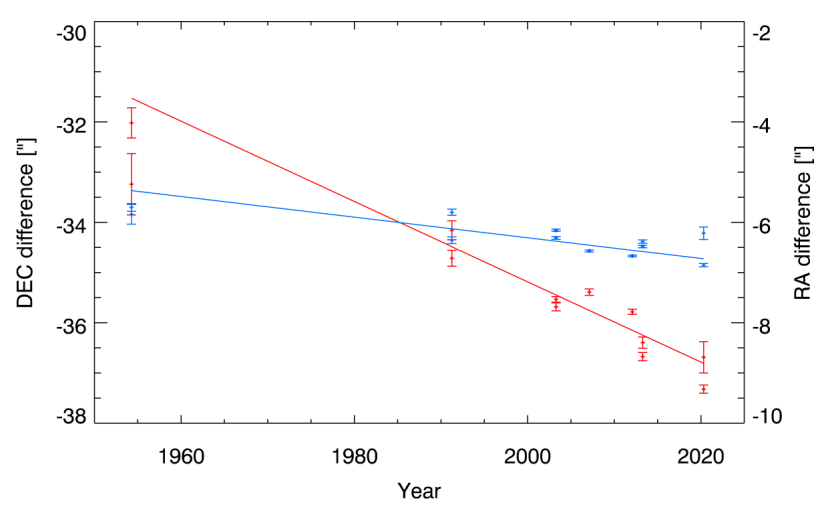

Fig. 7. HHO position displacement with regard to the ALLWISE position (Cutri et al. 2014) over time (red - Dec, blue - RA). The respective regression lines are shown as well.

r367497 obtained 2003, r1018994 and r1018997 obtained 2013) were retrieved from the IGAPS image server. These are supplemented by archival Tautenburg $\mathrm{H} \alpha$ images $(2007,2012)$ and two frames taken in 2020 with the new TAUKAM instrument (Stecklum et al. 2016). The POSS 1 and $2 R$-band images (1954, 1991) were added as well, using both plate digitisations from STSci and SuperCosmos. Before deriving the HHO positions by fitting its image profile, all frames were tied to the Gaia EDR3 astrometric reference system (Gaia Collaboration 2020).

The coordinate offsets of the HHO relative to the driving source for the various epochs are shown (Fig. 7), along with their respective linear fits. For the distance given above, the Dec slope (red) corresponds to a velocity of $-91.0 \pm 3.5 \mathrm{~km} \mathrm{~s}^{-1}$. Assuming constant speed, this implies a kinematic age of $\sim 430 \mathrm{yr}$. It is likely a major accretion event happened around that time that induced jet strengthening. Accounting for the position angle of the HHO movement of $190^{\circ}$ (measured from $\mathrm{N}$ through $\mathrm{E}$ ), a total PM speed of $92.4 \pm 3.5 \mathrm{~km} \mathrm{~s}^{-1}$ can be derived. With the help of an RV estimate of $-74.8 \pm 4.6 \mathrm{~km} \mathrm{~s}^{-1}$, obtained by lowresolution spectroscopy of the HHO using the Nasmyth spectrograph at the Tautenburg telescope, the inclination of the velocity vector follows as $51^{\circ} \pm 2^{\circ}$. This intermediate inclination is consistent with the cometary appearance of the YSO in the optical.

This example shows that the IGAPS $\mathrm{H} \alpha$ line emission images provide an excellent means for the detection of HHOs. In this instance, neither the driving YSO nor the HHO are detected even in the $i$ band, and yet the detection of the HHO in $\mathrm{H} \alpha$ is clear, at around 30 counts above background (depending on the seeing). Moreover, the 1 arcsec resolution of IGAPS images allows for good position measurements of these small (but extended) nebulous objects. Together with the availability of repeated IGAPS observations, with several years of epoch difference, precise PM measurements are possible, from which information about the physics of the HH flows, as well as their driving sources, can be obtained.

\section{Image mosaics}

The background of astronomical images would ideally be flat and dark. In reality the background in images from ground-based telescopes varies due to the interplay of different sources (e.g. airglow, moonlight) contributing varying levels of unwanted light.

To begin to tackle this securely, when working with the standard image data reduction available in the database, we recommend using the available confidence maps to reject pixels with confidence levels of less than 90-95\% typically. But we note that the level can vary across the sky and between filters, usually requiring a lower confidence level threshold for the $g$ filter and especially $U_{\mathrm{RGO}}$.

The MONTAGE software ${ }^{6}$ can be used to effectively mosaic images in a given filter, re-projecting images as needed to a single-projection algorithm and direction, adjusting the background levels in overlapping pairs of images to produce a smooth mosaic over a large area.

In addition to uniform background light sources, the $i$-band can sometimes be beset by fringing that originates from the airglow $\mathrm{OH}$ lines interfering via internal reflections in the CCD chips. This wave-like structure contributes $\sim 2 \%$ of the total counts in some images (Irwin \& Lewis 2001). It is almost entirely removed in the CASU pipeline (who use a library of $i$-band fringe frames from other INT WFC observing runs), although some will remain at the $\sim 0.2 \%$ level due to night-tonight variations. When $i$-band data are mosaicked, overlapping fringing is occasionally exaggerated and can remain visible in some mosaics.

As noted in Sect. 4, IPHAS observations were at first carried out at any level of moon brightness throughout the Galactic plane season. Observations during bright time were soon found to exhibit varying levels of background counts in the form of a small but noticeable gradient across each CCD (leading in later seasons to tighter moon phase and distance requirements). Ultimately, $\sim 8 \%$ of all IPHAS images were taken under such conditions.

Moonlight affected IPHAS images through both scattered light across the night sky and a component reflecting off the inside of the telescope dome and across the CCD array. The resulting illumination is therefore not necessarily uniform across all four CCDs, and requires a CCD-by-CCD solution. Its character is also influenced by the phase of the moon, its altitude above the horizon, angular separation from the pointing of the telescope, and the extent (and position) of cloud cover across the sky. These relatively small gradients can be exacerbated by mosaicking CCD images over large areas of the sky (many degrees), becoming a significant issue in the production of large mosaics.

The recommended solution to removing the moonlight and achieving a flat and dark background is to model and fit the background gradient for each CCD. Since the $r$ and $\mathrm{H} \alpha$ band images contain nebulosity that could affect the fit, we recommend fitting the background gradient to $r-H \alpha$ images (after scaling the images to correct for their different exposure times), since both filters contain the $\mathrm{H} \alpha$ and forbidden [NII] lines that typically dominate diffuse astronomical emission. Binning the image into $100 \times 100$ pixel bins and taking the median pixel value in each bin provides a simple method to measure the background level in that bin. A two-dimensional gradient of the form $Z=A x+B y+C$ can then be fit to the data, where $A, B$ and $C$ are free parameters, using, for example a Markov chain Monte Carlo simulation and the Python code emcee (Foreman-Mackey et al. 2013). More complex models have been tested (including Fourier transform techniques), but none were found to provide a significant improvement. In short, the two-dimensional gradient method will prove effective in the majority of cases.

Some care should also be applied when very bright stars fall on (or near) one of the CCDs, as saturation, atmospheric and lens effects can heavily affect an image and the model fit to it. Identifying and excluding a magnitude-dependent radius around

6 Available from http://montage.ipac.caltech.edu/ 


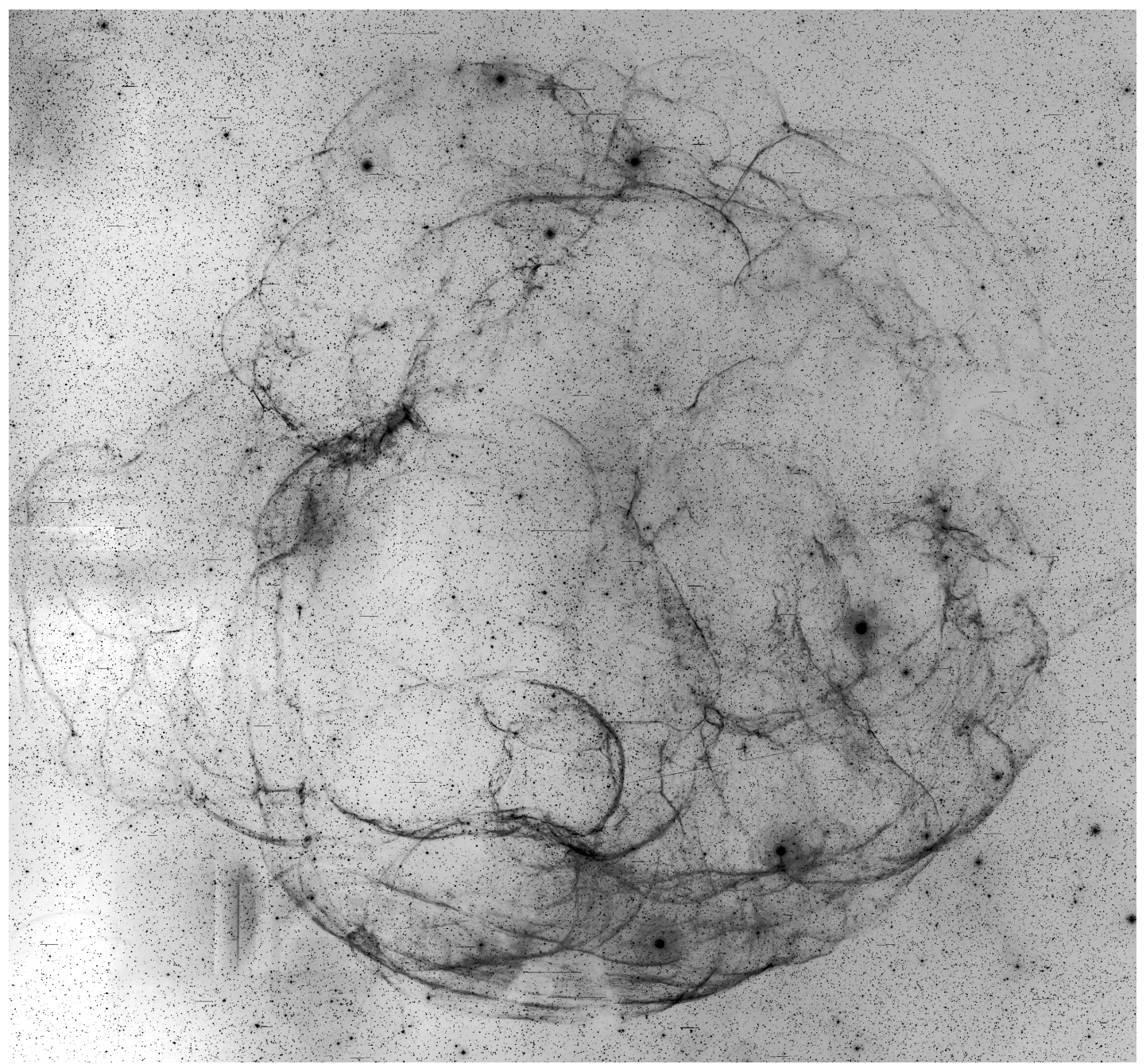

Fig. 8. SNR S147. This is a full resolution background-corrected mosaic made from the H $\alpha$-filter data (no $r$ subtraction). Approximate image dimensions are $4.2 \times 3.6$ sq. deg. North is up, and east is to the left. The grey scale is negative such that the brightest emission is darkest. Compared to the earlier mosaic appearing as Fig. 19 in Drew et al. (2005), based on $\mathrm{H} \alpha-r$ data, with background fitting and removal, there are fewer artefacts thanks in large part to the incorporation of better re-observations. Much of the faint structure left, such as the ragged diffuse emission in and below the centre of the remnant, is real.

such stars using the Tycho-2 catalogue of bright stars (Høg et al. 2000) proved effective for overcoming these problems.

As an example of the potential of IGAPS image mosaicking, we present a large, $4.2 \times 3.6$ degree $\mathrm{H} \alpha$ mosaic of the SNR Simeis 147, produced using the techniques described above. Simeis 147 (hereafter S147) is otherwise known as SNR G180.0-01.7, Shajn 147, or Sharpless 2-240. It is a large, faint, late-stage remnant located just below the Galactic anticentre. It was discovered in 1952 and lies at a distance of $0.8-1.6 \mathrm{kpc}$ (Gvaramadze 2006), on the near side of the Perseus spiral arm. The SNR consists of numerous filaments embedded in large-scale diffuse emission. The eastern and western edges of S147 show signs of blowouts, the southern edge shows a sharp boundary, with a less regular one in the north. The undistorted appearance of the SNR may partly be due to it expanding into a region of space already partly cleared by a previous supernova (Gvaramadze 2006). Regardless, examples of large and pristine SNRs are rare and observations of them can be important for constraining hydrodynamic simulations of their expansion and structure.

Figure 8 shows the full mosaic constructed as outlined above. The challenge of this object is its great size, allied with very intricate and sometimes very faint small-scale detail. The num- ber of individual CCD frames included is in the region of 250 . The full-resolution S147 mosaic (199 MB) itself is provided as a fits-formatted file which can be downloaded from the IGAPS website.

\section{Nebular target selection for massive-multiplex spectroscopy}

The next decade will see an increase in large, multi-object digital spectroscopic surveys on 4 m class telescopes. In drawing up target lists, these surveys will make use of the data that has been acquired by wide-area digital photometric surveys, like IGAPS. Two examples due to start soon are the WEAVE survey on the 4-m William Herschel Telescope (WHT) of the Roque de los Muchachos Observatory in La Palma (Dalton et al. 2020) and the 4MOST survey on the 4-m VISTA telescope operated by ESO at Paranal (de Jong et al. 2019). Both facilities will collect of the order of 1000 targets per pointing, and a major science driver for both is Milky Way science in the Gaia era.

Massive-multiplex spectroscopy requires informed target selection. The IPHAS H $\alpha$ images, in particular, can characterise the diffuse sky for studies of the ionised ISM. Here, we outline 
a software method - named HAGRID - aimed at doing this through the interrogation of $\mathrm{H} \alpha$ images. The positions generated by HAGRID will be used in constructing the SCIP ('Stellar, Circumstellar and Interstellar Physics') northern Galactic plane programme - a strand within the overall WEAVE 5-year survey. The software is also being deployed to find targets for the southern plane (based on VPHAS + data), to enable similar observations via 4MOST. In the text below, the acronym WEAVE appearing on its own will stand both for the instrument and for the WEAVE/SCIP survey strand, according to context.

\subsection{Building lists of science targets with $H_{\mathrm{A}} G_{\mathrm{RID}}$}

A WEAVE pointing is defined by the coordinates of its centre and a field of view of radius of 1 degree, projecting a circle on the sky covering $\pi$ sq. deg. Within such a field, the aim is to identify several hundred positions that coincide with regions of (locally) maximum $\mathrm{H} \alpha$ brightness.

In outline, the steps taken are as follows: (i) find all $\mathrm{H} \alpha \mathrm{CCD}$ images from the IGAPS repository in the area of interest; (ii) mask out stars, CCD borders, bad pixels, and vignetted areas from the data; (iii) divide the image into superpixels; and (iv) select the superpixels with the highest counts as candidate source positions.

The application of the algorithm, confronted with real data, is necessarily more complicated, particularly as it must deal as far as possible with all the artefacts that mimic real nebulosity. So we now itemise the steps involved in more detail.

The first step is to collect the CCD images from the IGAPS repository in the area of interest. For one WEAVE field of radius 1 degree, this will be a list of over $100 \mathrm{H} \alpha$ CCD frames and their associated $r_{I}$ exposures. Since frames with poor data quality often lead to false detections of $\mathrm{H} \alpha$ emission, we excluded, where possible, grade C and D frames and favoured uniformly calibrated over pipeline-calibrated data.

The second is to create a star mask for each CCD image from both the IGAPS point-source database and a bright star catalogue to identify stars needing larger exclusion zones. The mask radius of IGAPS sources is set as a function of $r_{I}$ magnitude, $\mathrm{H} \alpha$ seeing and ellipticity. Also masked are diffraction spikes of bright stars and a visible halo for very bright stars $(<4.5 \mathrm{mag}$; see the right panel of Fig. 9). The halo position relative to the star depends on the angular separation of the star from the optical axis of the telescope.

Third, other artefacts are also masked. These include CCD borders, pixels that fall below a specified threshold in the linked confidence map (see Appendix A.2), hot or cold pixels, and artificial linear structures (satellite trails, noise bands, gain-change strips, bright star reflections, etc.) found by visual inspection.

The fourth step is to create superpixels and rank them by $\mathrm{H} \alpha$ brightness. Each masked CCD frame is divided into superpixels, which are squares of $n \times n$ native pixels, where $n$ is an adjustable input parameter. For WEAVE, $n=25$ giving $8.25 \times 8.25 \operatorname{arcsec}^{2}$ superpixels. This choice tensions between good-enough angular resolution and the typical loss of area and statistics inflicted by the masking. Superpixels that are more than 50\% masked are rejected. To avoid particle hits being mistaken for astronomical signal, the data are median filtered using $3 \times 3$ pixel (about $1 \operatorname{arcsec}^{2}$ ) binning. The superpixels are then ranked by mean count determined from the unmasked pixels.

The fifth is to estimate the $\mathrm{H} \alpha$ sky background. A sound determination of the local sky value is very important, especially for determining the correct $\mathrm{H} \alpha$ surface brightness. The algorithm measures the sum of sky and any significant astronomical back-

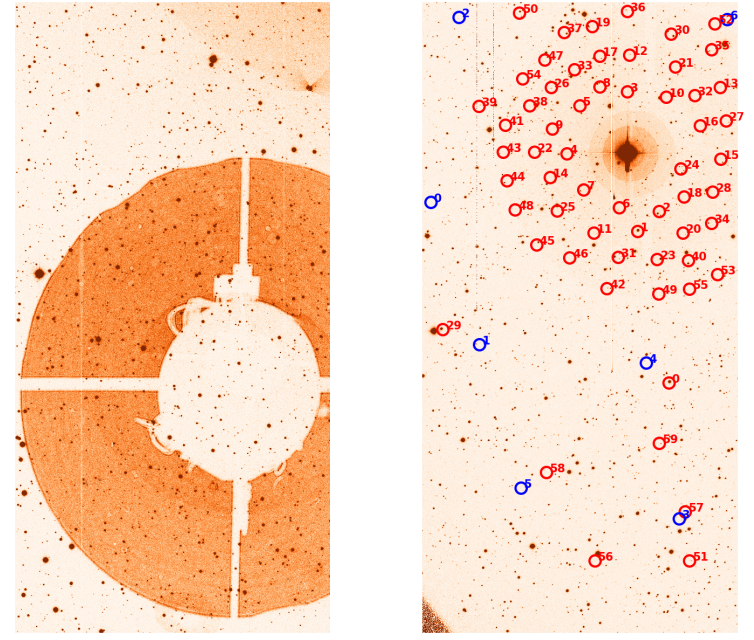

Fig. 9. Two examples of common reflected light artefacts in IGAPS imagery. Left: ghost on image r372056, CCD\#4, due to a bright star. We see that the stray-light image even picks up details of protruding cabling at the prime focus of the INT. Part of a much fainter ghost can be seen at the top right. Right: example of a halo around a bright star fooling the HAGRID selection from r541554, CCD\#3. Source positions selected by HAGRID are shown as red numbered circles. They crowd into the faint extended halo that is a little offset from the star position. A larger stellar mask for bright stars and the rejection of 'star-like' selections as described in Sect. 7.2 helps eliminate these. The blue circles mark identified count minima that are stored as potential sky fibre positions.

ground from the $\mathrm{H} \alpha$ frames. In predefined areas of extensive and intense nebulosity we also derive an estimate of the $\mathrm{H} \alpha$ sky-only background value from the $r_{I}$ sky value. This uses a linear fit to a global plot of the $\mathrm{H} \alpha$ against the $r_{I}$ sky level, exploiting the fact that most of the Galactic plane is free from nebular emission. If the sky value inferred from $r_{I}$ is lower than the $\mathrm{H} \alpha$ sky value, then we adopt the average of the two. Taking the average was precautionary against problems with the $r_{I}$ sky prediction due to changing moonlight reflections into the telescope from clouds and other structures such as the dome.

The sixth is to select $\mathrm{H} \alpha$-excess source positions from the ranked superpixel list for every CCD frame. The superpixel list is searched starting at the highest mean count. A superpixel is rejected if: it is closer than a distance limit $(1 \mathrm{arcmin}$ for WEAVE) to an already selected superpixel; the difference between the superpixel mean and the frame $\mathrm{H} \alpha$ sky is less than the sky noise (one sigma). Finally, the maximum $3 \times 3$ pixel mean-filtered count within the superpixel is located and its position is adopted as the candidate target position. If the difference between this more localised mean and the $\mathrm{H} \alpha$ sky is $>10 \mathrm{ADU}$, the superpixel goes forward into a merged overview table.

Seventh, the location, count, surface brightness, and other data for each selected high-H $\alpha$ candidate position is appended to the overview table, which covers a large user-defined sky area that is ready for further checking and analysis.

As it searches for positions of bright $\mathrm{H} \alpha$ emission, HAGRID also identifies suitable low-count sky positions and gathers statistics on sky noise. The distribution of sky noise versus sky background it finds closely resembles the distribution found by the pipeline shown in Fig. 3. The sky noise measured by HAGRID is lower as it does not involve a fit over the whole CCD and so avoids contributions from fainter stars.

The algorithm can be applied to areas of arbitrary size. As each CCD is independent of the others, the code parallelises very 


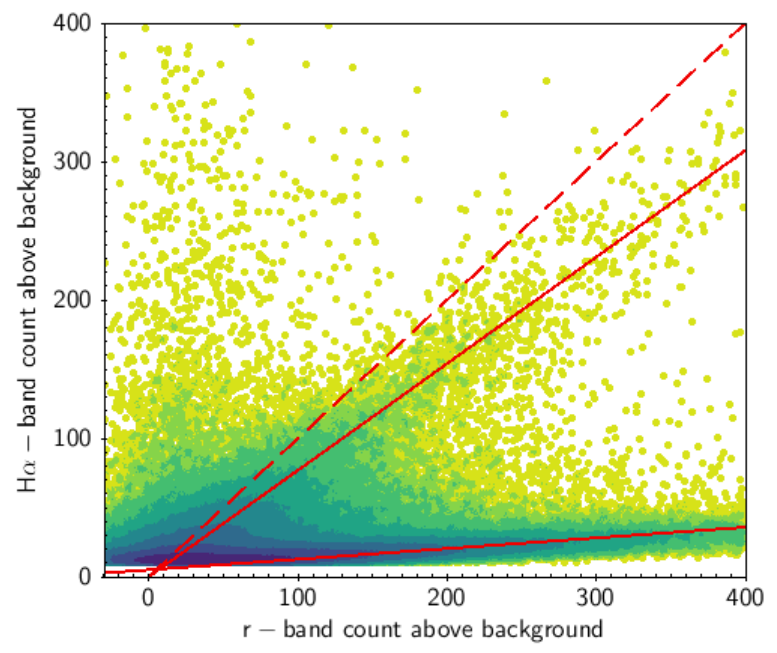

Fig. 10. Excess $\mathrm{H} \alpha$-band counts compared with the excess counts in the $r_{I}$ band. Both quantities are the difference between the measured peak count and the estimated background level returned by HAGRID. It should be noted that the HAGRID output list is restricted to excess narrow-band counts $>10$. The data are plotted as a density map and before any second-stage cleaning. The selection shown is from the sky region: $\left(30^{\circ}<\ell<95^{\circ},|b|<4^{\circ}\right)$. Working from top to bottom the red lines are: equality between counts (shown dashed), a line of slope 0.77 (solid) representative of nebula-dominated positions, and a line of slope $1 / 13$ (solid) characteristic of star-like positions.

effectively. The choices of minimum distance between accepted source positions is driven mainly by the design of the destination wide-field spectrograph, and the anticipated observing strategy. For WEAVE, applying the 1 arcmin minimum distance between fibre placements led to at most $\sim 200$ source positions selected per WFC CCD (with a much lower median of 5).

\subsection{Final processing: List cleaning and reduction}

The list of potential target positions generated for WEAVE as described above was long and needed further cleaning and reduction. Not everything that appears bright in $\mathrm{H} \alpha$ and is passed through by HAGRID has an astronomical origin: for example, some satellite trails, ghosts and unrecognised haloes around brighter stars can remain (Fig. 9). There are a number of further test-and-eliminate steps that can be taken to reduce the list to a high-confidence core. One such step is to favour repeat selection of the same emission structure and to reject isolated points (typically due to cosmic ray strikes that slip through). Generally speaking, we expect any given high-surface-brightness structure to be picked up twice or more, given that most sky locations are covered by a minimum of two images.

An important piece of empirically driven post-analysis is illustrated by Fig. 10, which compares the excess $\mathrm{H} \alpha$ counts above the estimated background level with the excess obtained in the $r$ band, after scaling the latter to correct for the shorter exposure time. Two main trends, drawn as solid lines, are apparent. The uppermost of the two runs a bit below the equality line. In the ideal case where $\mathrm{H} \alpha$ and [NII] $654.8,658.4 \mathrm{~nm}$ nebular line emission dominate the total counts measured in the $r$ band, the expectation would be that the measured count excesses in both the narrow and the broad band would be the same (given that the peak transmission in the narrow-band filter corrected for CCD response is closely comparable to the mean of the same quantity for the $r$ band). This ideal does not apply,

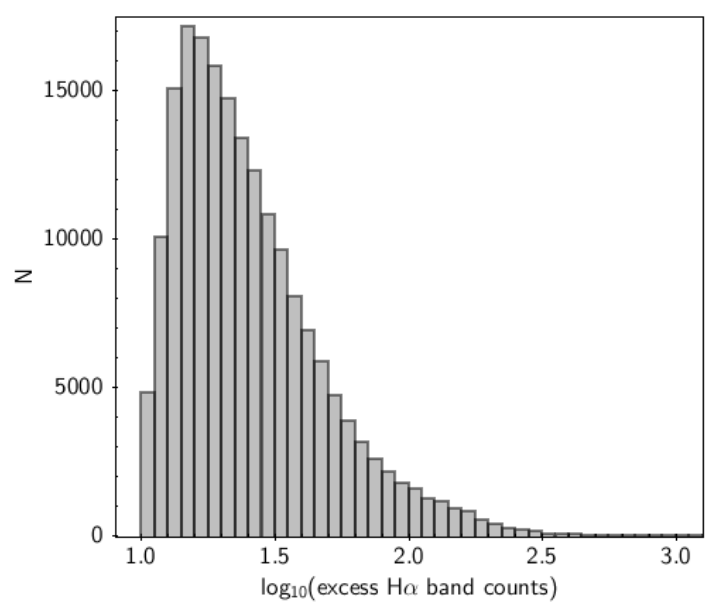

Fig. 11. Final distribution of selected diffuse ISM sky positions for the WEAVE footprint as a function of the logarithm of excess $\mathrm{H} \alpha$ counts. It is highly skewed to low excess counts. A rough translation into surface brightness is that ten excess counts, the minimum accepted, corresponds to $\sim 3 \times 10^{-16} \mathrm{ergs} \mathrm{cm}^{-2} \mathrm{~s}^{-1} \operatorname{arcsec}^{-2}$.

because of other nebular lines within the $r$ band, not captured by the narrow band (e.g. the [SII] 671.6, 673.1 nm doublet that can be strengthened by shock excitation, and potentially some [OI] 630.0, 636.2 nm emission). The approximate regression line shown in Fig. 10 has a slope less than 1, for this reason. The objects of the search are indeed the candidate positions clustered around this empirical trend and, as such, they are the ones to keep.

In contrast, the second much lower gradient trend apparent in Fig. 10, running close to the horizontal axis, is created by sky locations where the spectrum is continuum-dominated (i.e. starlike). These locations can be stellar haloes where HAGRID picks up a seeming $\mathrm{H} \alpha$ excess thanks to the typically wider seeing profile in the longer and unguided narrow-band exposures, or ghosts (see Fig. 9 for examples). In the case of a typical $0.5 \lesssim r-i \lesssim 1$ stellar continuum across the $r$ band, the expectation would be that the narrow-band excess counts would be approximately $1 / 13$ of the $r$ counts - this is the last of the three lines superimposed in Fig. 10. Candidate positions of this type need to be removed.

To make an accept/reject decision for every candidate position in the list, the distances to the expected nebular and stellar trend lines are calculated. These distances, $N$, are then expressed scaled to $\sigma$, the relevant Poisson-like error on the computed distance (subscript $n$ for nebular, $s$ for star-like). This is followed by cuts applied in the $N_{\mathrm{n}}, N_{\mathrm{s}}$ plane to select the most credible nebular targets. Inevitably, at low count levels, the confidence in assigning a candidate to the 'nebular' and 'star-like' classes weakens greatly. The minimum excess count of 10 imposed by HAGRID helps deal with this, but a minimum cut on $N_{\mathrm{s}}$ is also needed. Where it is placed has to be tested empirically: For WEAVE we required $N_{\mathrm{s}}>3.5$.

The selection can also be trimmed down to surface densities appropriate to the instrument used and the survey observing strategy (e.g. number of visits, required science sampling). In the case of WEAVE this meant a 2 arcmin grid was placed over the relevant sky area and only two positions with the highest flux were kept in each grid cell. Taking all the steps together for the WEAVE example, the original list of about 1.3 million target positions reduced to under 200000 potential targets, of which we expect around one-fourth to be selected for observing. 

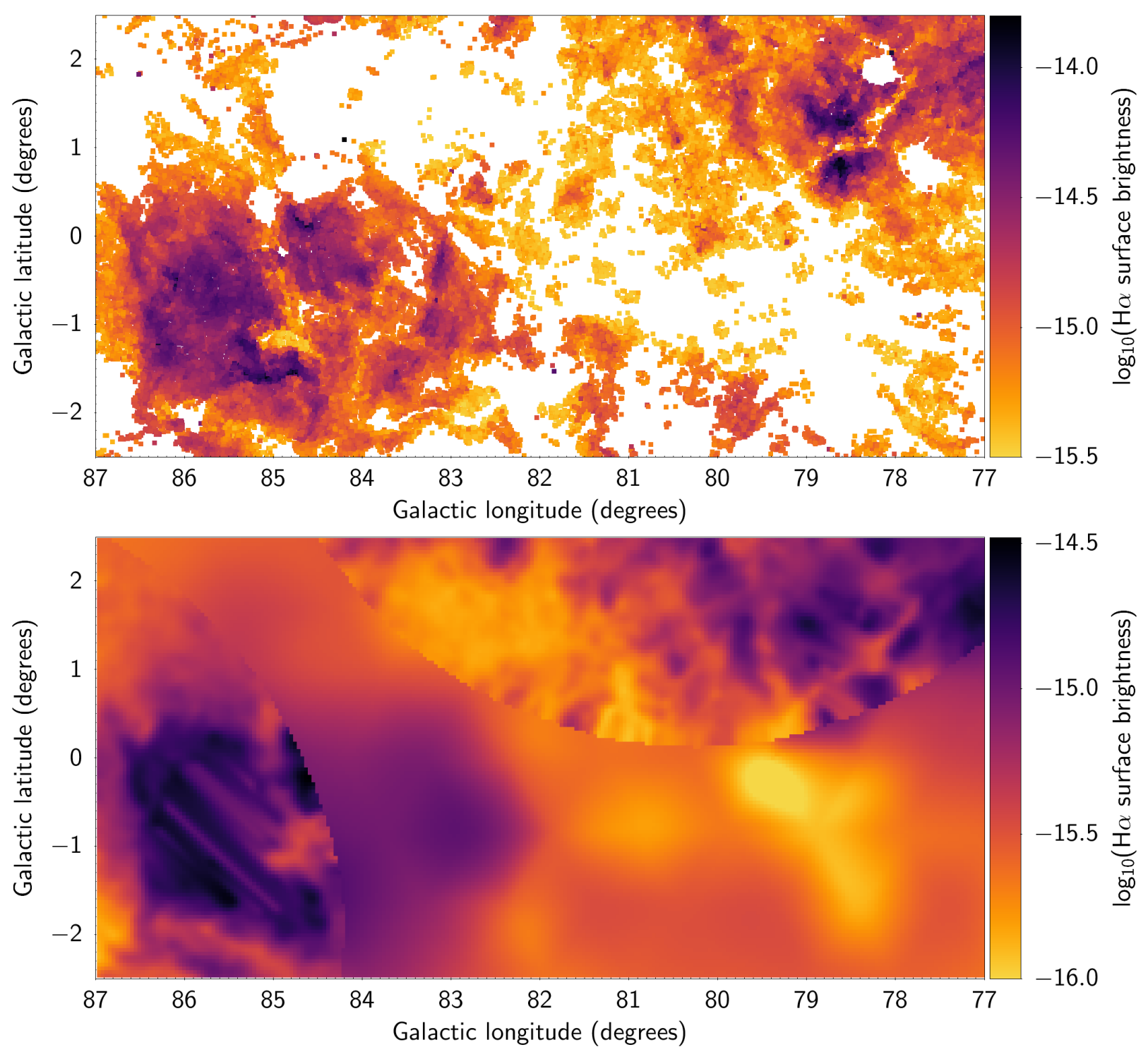

Fig. 12. Comparison between HAGRID generated flux levels and the calibrated map of Finkbeiner (2003). Top: map of HAGRID-selected positions in the Cygnus-X region of the northern Galactic plane. Every selected position has been coloured according to the logarithm of the estimated surface brightness (in ergs $\mathrm{cm}^{-2} \mathrm{~s}^{-1} \operatorname{arcsec}^{-2}$ ). White areas will have $\log$ (surface brightness) $<-15.5$. The region shown spans 50 sq. deg., or $3.6 \%$ of the total area processed, and contains $20 \%$ of the identified candidate target positions. It is the most line emission-rich part of the northern plane. Bottom: same area in the Finkbeiner (2003) $\mathrm{H} \alpha$ map, with 6 arcmin pixels. The data available in this region are a combination of images from VTSS (circular footprint) and WHAM (making up the much coarser resolution background).

\subsection{Testing the downsize against known Herbig-Haro objects}

We performed a retrospective test that compared the character of the long list with that of the final downsize by cross-matching them both with a list of known HHOs. This list was established by a CDS criteria query using the term otype=' HH' \&ra $>0$. The coordinate condition was necessary to dismiss about 800 entries without coordinate information. The resulting table comprised 2622 positions: just 388 of them lie in the sky region defined by the Galactic coordinate ranges, $30^{\circ}<\ell<210^{\circ}$, $|b|<4^{\circ}$ (roughly the footprint HAGRID has been applied to). When a limit of 10 arcsec was set on the angular separation, there were 70 and 40 successful cross-matches with the HAGRID long and short lists, respectively. These numbers dropped to 45 and 27 when the limit on angular separation was reduced to 5 arcsec.
That no more than $20 \%$, at best, of the listed HHO in the region were recovered is attributable to $\mathrm{HHO}$ position uncertainties, their high PM and the relatively low surface brightness of many. Another occasional factor at work will be the presence of substantial scattered starlight lowering the contrast between the $\mathrm{H} \alpha$ and $r$ images to below an acceptance threshold (emission in the vicinity of V645 Cyg is subject to this). The most relevant point is that the down-sized list of candidate emission line positions captured more than half the number matching with the long list, despite the fact it contained only $\sim 0.15$ as many positions. Proportionately, the shorter list was appreciably better, indicating that the downsizing had the side benefit of raising list quality.

\subsection{Results of selection}

The distribution of diffuse ionised emission in the northern plane is heavily weighted to low surface brightness and its presence 


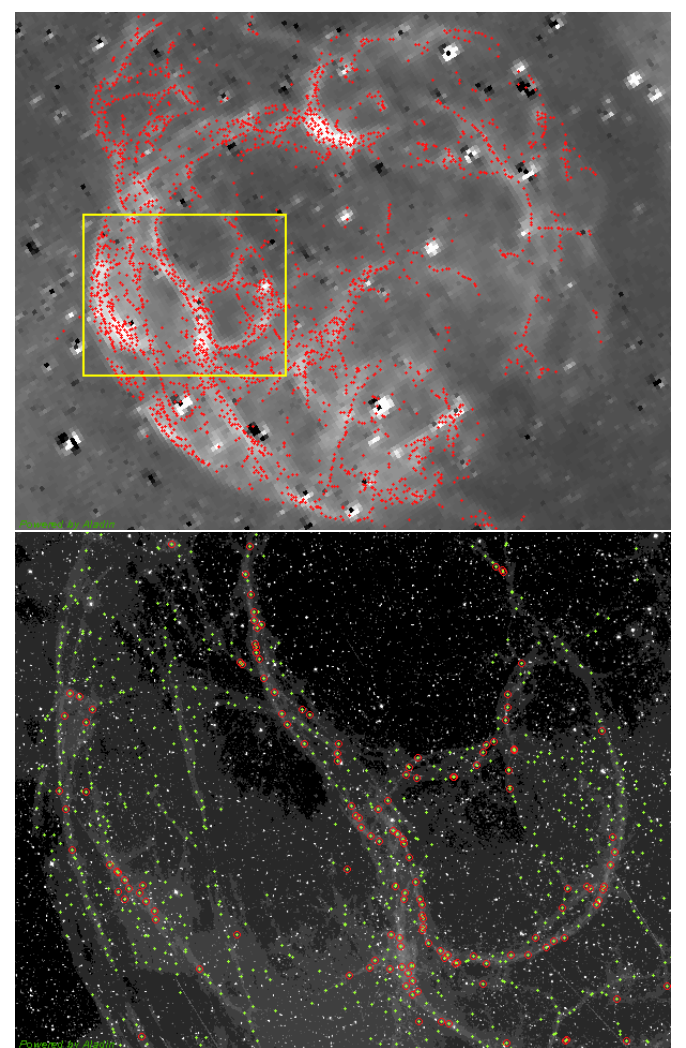

Fig. 13. S147, the SNR, as a test of HAGRID position selection. Upper panel: comparison between the pattern of final HAGRID positions, in red, and the VTSS (Dennison et al. 1998) arbitrarily scaled continuumsubtracted $\mathrm{H} \alpha$ surface brightness map shown in grey scale. A good correspondence is achieved. Lower panel: zoom into a $0.27 \times 1.00 \mathrm{sq}$. deg. area within the remnant superposing HAGRID positions on the IGAPS $\mathrm{H} \alpha$ mosaic at full $\sim 1$ arcsec resolution (shown in full in Fig. 8). This shows how the fine filaments making up the remnant are accurately traced by the HAGRID selections (green dots): Those with surface brightness exceeding $10^{-15} \mathrm{erg} \mathrm{cm}^{-2} \mathrm{~s}^{-1} \operatorname{arcsec}^{-2}$ are encircled in red. Both panels use Galactic coordinates such that latitude increases upwards and longitude towards the left.

along the plane is extremely uneven. These are the outstanding features of the out turn from the application of HAGRID to the IGAPS image database. The extent to which low surface brightness is favoured is illustrated by the histogram of excess $\mathrm{H} \alpha$ counts, presented as Fig. 11. The mean of the distribution is 32.5 excess counts, while the more informative median is just 21.6 (translating to a surface brightness of $\sim 7.2 \times$ $10^{-16} \mathrm{ergs} \mathrm{cm}^{-2} \mathrm{~s}^{-1} \operatorname{arcsec}^{-2}$ or close on 130 rayleighs).

The upper panel of Fig. 12 is a cut-out of $50 \mathrm{sq}$. deg. in the Cygnus- $X$ region of the plane, a region that is very rich in ionised gas: around $20 \%$ of all the selections are contained in just this $3.6 \%$ of the processed sky area. Every data point is a candidate target position and is coloured according to the logarithm of $\mathrm{H} \alpha$ narrow-band surface brightness. The figure amounts to a 'pointilliste' rendition of an $\mathrm{H} \alpha$ image of the region emphasising the brightest locations. Comparison with the much coarser angular resolution $\mathrm{H} \alpha$ map presented by Finkbeiner (2003), in the lower panel of Fig. 12, shows that the larger-scale structure in the $\mathrm{H} \alpha$ surface brightness is shared, with the important difference that the HAGRID output offers much better resolved, brighter detail that vanishes when smoothed to several arcminutes. The bright North America Nebula is located towards bottom left in both panels. The hole in the emission in the top right corner of the upper panel is a product of needing to leave a zone clear around the 2nd magnitude star, $\gamma$ Cyg. The larger hole below it is real in the sense that it is a dark cloud free of line emission.

A further example of the candidate positions output by HAGRID and the subsequent reduction is presented in Fig. 13 for the case of S147 (already discussed and illustrated in Sect. 6). Its appearance is very filamentary, somewhat resembling a collection of soap bubbles, with the filaments at the interfaces. The overall distribution of emission is captured very well by the selection, as shown by the comparison with the excerpt from the Virginia Tech Spectral-line Survey (VTSS, Dennison et al. 1998), in the upper panel of Fig. 13. However, the 1.6 arcmin pixel scale of VTSS does not entirely resolve the structure present. The filaments, with typical widths of under 1 arcmin, emerge more clearly in the IGAPS narrow-band imagery, thanks to its native $\sim 1$ arcsec angular resolution. This is picked up faithfully by the HAGRID selection. The surface brightness of the selections ranges from $3 \times 10^{-16}$ to (very infrequently) $3 \times 10^{-15} \mathrm{ergs} \mathrm{cm}^{-2} \mathrm{~s}^{-1} \operatorname{arcsec}^{-2}$.

\section{Closing remarks}

A goal of this paper has been to present and describe the new IGAPS image database, formed from merging the data from the IPHAS (Drew et al. 2005) and UVEX (Groot et al. 2009) surveys of the northern Galactic plane. Around two-thirds of the database carries photometric zero points from the uniform calibration described previously by Monguió et al. (2020). The collection is complete in that it contains all images of all qualities obtained over the course of the two long-running survey programmes. This creates options for comparing different epochs given that many fields were observed more than once.

The main focus of this paper has been on the $\mathrm{H} \alpha$ narrow band: the only one of the IGAPS set expressly targeting line emission. Before summarising what we have presented on $\mathrm{H} \alpha$, we recall that the Sloan $g$ band contains within its range the sometimes extremely bright [O III] 495.9, $500.7 \mathrm{~nm}$ doublet. Accordingly, images taken using this filter can be used to compare and contrast the appearance of prominent nebulous regions in low ionisation lines ( $\mathrm{H} \alpha$ and the [N II] 654.8, $658.4 \mathrm{~nm}$ doublet) and the much higher ionisation [O III]. This will work especially well for lower extinction sightlines, where the [O III] lines are not disadvantaged by dust obscuration on top of the 1:4 exposure time ratio. An outstanding example of such a comparison, for the Dumbbell Nebula, is shown as Fig. 14. This is a large and bright PN in which it can be seen that the [O III] emission ( $g$-band image, upper panel) is less clumpy than the $\mathrm{H} \alpha+[\mathrm{NII}]$ emission ( $\mathrm{H} \alpha$ image, lower panel), while the extent of the main nebula is nearly the same in both. Furthermore, even in the $g$ band 30-s exposure, fainter more extended structure is also apparent beyond the main nebula rim (seen to the west, i.e. to the right in the figure). Its existence was first noticed in the lower-resolution narrow-band images presented by Papamastorakis et al. (1993). The central star also stands out at this shorter wavelength. The price paid for this kind of direct exploitation is, necessarily, the strong pickup of starlight in the field because the $g$ band is broad. Some improvement on this might be achieved by constructing $g-r$ difference images.

Returning to our main aim, we have provided a characterisation of the distinctive narrow-band $\mathrm{H} \alpha$ data whilst also showcasing some new illustrative applications. Thanks to the Monguió et al. (2020) zero point calibration, it is now more certain what the noise levels and sensitivities are: At full $\sim 1$ arcsec angular resolution it is possible to distinguish the nebulosity of 


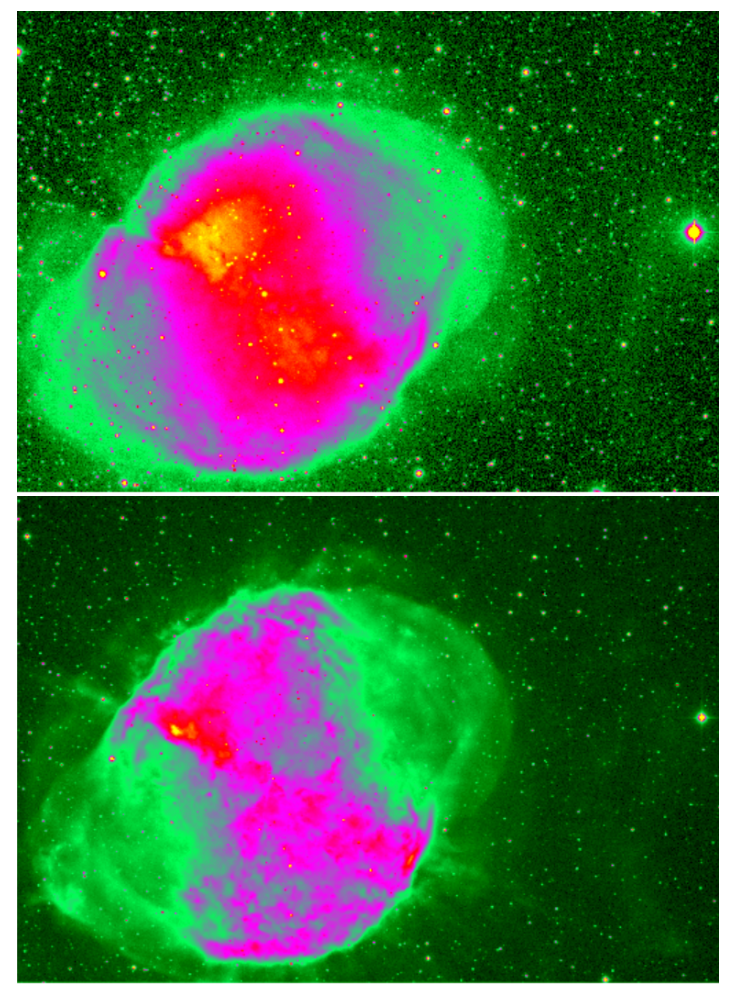

Fig. 14. Dumbbell Nebula: as imaged in the $g$ and $\mathrm{H} \alpha$ bands (upper and lower panels, respectively). The $g$ image includes the strong [O III] $495.9,500.7 \mathrm{~nm}$ lines. The cutouts are taken from the same corner of CCD \#2 in runs r-477762 and r-1241402, picked for having wellmatched seeing (respectively 1.31 and 1.25 arcsec). The colour scales used are capped at 1250 counts for $g$ and 5000 counts for $\mathrm{H} \alpha$ to match the ratio of their exposure times. They run from black at background level up through green/magenta/red to yellow at the bright end. This PN has a diameter of approximately 6 arcmin. North is up, east to the left.

surface brightness down to $\sim 2 \times 10^{-16} \mathrm{ergs} \mathrm{cm}^{-2} \mathrm{~s}^{-1} \operatorname{arcsec}^{-2}$ (the typical noise level). We have described here, in detail, how this may be exploited on a large scale to build target lists for diffuseISM spectroscopy using the coming generation of massivemultiplex wide-field spectrographs (Sect. 7 on the HAGRID algorithm). On re-binning the native 0.333 arcsec pixels, the sensitivity increases as expected: Sabin et al. (2014) already claimed a typical sensitivity of $\sim 10^{-17} \mathrm{ergs} \mathrm{cm}^{-2} \mathrm{~s}^{-1} \operatorname{arcsec}^{-2}$ for $5 \times 5 \operatorname{arcsec}^{2}$ binning. We endorse this, with the necessary qualification that, in reality, there will always be a range in sensitivity, linked directly to the prevailing background level (see Fig. 3).

So far, most science exploitation of IGAPS image data has been directed towards PNe and, to a lesser extent, SNRs. We anticipate this will continue in upcoming programmes using WEAVE and other new generation wide-field spectroscopic instruments. But there is also the opportunity to use the $\mathrm{H} \alpha$ images in particular to support science exploration of the diffuse ISM in star-forming regions. In particular, the software tool HAGRID permits dense sampling across a wide area of many of the HII regions of the northern plane (as illustrated by Fig. 12). Target lists of this kind for multi-object fibre spectroscopy will be included in the WEAVE survey and should lead to new insights into the detailed chemistry and kinematics of the diffuse environment in and around young star clusters. A programme of a similar kind is already underway using LAMOST (Wu et al. 2020): It samples the northern plane from a catalogue also built with the assistance of IPHAS images, now contained within the database presented here.
The full collection of IGAPS images is available to the community online ${ }^{7}$. This website provides an interface that facilitates downloads of selected images, served up as individual CCD frames. It also provides a number of related resources, including the HAGRID-generated list of bright diffuse $\mathrm{H} \alpha$ northern-plane positions presented here.

Acknowledgements. This work is based on observations made with the Isaac Newton Telescope operated on the island of La Palma by the Isaac Newton Group of Telescopes in the Spanish Observatorio del Roque de los Muchachos of the Instituto de Astrofísica de Canarias. This research has made use of the University of Hertfordshire high-performance computing facility (https: //uhhpc.herts . ac .uk/) located at the University of Hertfordshire (supported by STFC grants including ST/P000096/1). This study has used part of an image obtained by the Virginia Tech Spectral-Line Survey, which is supported by the National Science Foundation. We thank the following Bristol University students: Greg Mould, William Howie, Luke Davies, Heidi Naumann, Will Summers, Alex Townshend, Paul May, Matina Mitchell, Finn Hoolahan, Tom Burgess, Ashley Akerman, James Jordan, Simon Palmer, Anna Kovacevic, Jai Tailor, Olivia Smedley and Daniel Huggins for their work searching through difference images looking for PN candidates as part of their final year undergraduate projects. RG benefitted from support via STFC grant ST/M001334/1 as a visitor to UCL. JED and MM acknowledge the support of research grants funded by the Science, Technology and Facilities Council of the UK (STFC, grants ST/M001008/1 and ST/J001333/1). MM was partially supported by the MINECO (Spanish Ministry of Economy) through grant ESP2016-80079-C2-1$\mathrm{R}$ and RTI2018-095076-B-C21 (MINECO/FEDER, UE), and MDM-2014-0369 of ICCUB (Unidad de Excelencia 'María de Maeztu'). AM acknowledges support from the State Research Agency (AEI) of the Spanish Ministry of Science, Innovation and Universities (MCIU) and the European Regional Development Fund (FEDER) under grant AYA2017-83383-P. PJG is partially supported by NRF-SARChI grant 111692 and acknowledges support from the Netherlands Organisation for Scientific Research (NWO), in contributing to the Isaac Newton Group of Telescopes and through grant 614.000.601. Aspects of the analysis presented have been carried out via TOPCAT and STILTS (Taylor 2006). This research has made use of both the SIMBAD database and the "Aladin sky atlas", respectively operated and developed at CDS, Strasbourg, France. This research has also made use of the image manipulation software, MONTAGE. It is funded by the National Science Foundation under Grant Number ACI-1440620, and was previously funded by the National Aeronautics and Space Administration's Earth Science Technology Office, Computation Technologies Project, under Cooperative Agreement Number NCC5-626 between NASA and the California Institute of Technology.

\section{References}

Alam, S., Albareti, F. D., Allende Prieto, C., et al. 2015, ApJS, 219, 12 Anders, F., Khalatyan, A., Chiappini, C., et al. 2019, A\&A, 628, A94 Awang Iskandar, D. N. F., Zijlstra, A. A., McDonald, I., et al. 2020, Galaxies, 8, 88

Barentsen, G., Farnhill, H. J., Drew, J. E., et al. 2014, MNRAS, 444, 3230

Chambers, K. C., Magnier, E. A., Metcalfe, N., et al. 2016, ArXiv e-prints [arXiv:1612.05560]

Connelley, M. S., Reipurth, B., \& Tokunaga, A. T. 2008, AJ, 135, 2496

Corradi, R. L. M., Sabin, L., Miszalski, B., et al. 2011, MNRAS, 410, 1349

Cutri, R. M., Wright, E. L., Conrow, T., et al. 2014, VizieR Online Data Catalog: II/328

Dalton, G., Trager, S., Abrams, D. C., et al. 2020, SPIE Conf. Ser., 11447, 1144714

de Jong, R. S., Agertz, O., Berbel, A. A., et al. 2019, The Messenger, 175, 3

Dennison, B., Simonetti, J. H., \& Topasna, G. A. 1998, PASA, 15, 147

Dharmawardena, T. E., Barlow, M. J., Drew, J. E., et al. 2021, MNRAS, 501, 6156

Doi, M., Tanaka, M., Fukugita, M., et al. 2010, AJ, 139, 1628

Drew, J. E., Greimel, R., Irwin, M. J., et al. 2005, MNRAS, 362, 753

Drew, J. E., Gonzalez-Solares, E., Greimel, R., et al. 2014, MNRAS, 440, 2036

Farnhill, H. J., Drew, J. E., Barentsen, G., \& González-Solares, E. A. 2016, MNRAS, 457, 642

Fernández-Martín, A. 2007, Master Thesis on Astrophysics, La Laguna, University, Spain

Finkbeiner, D. P. 2003, ApJS, 146, 407

Foreman-Mackey, D., Hogg, D. W., Lang, D., \& Goodman, J. 2013, PASP, 125, 306

7 http://wWw.igapsimages.org 
Freyhammer, L. M., Andersen, M. I., Arentoft, T., Sterken, C., \& Nørregaard, P. 2001, Exp. Astron., 12, 147

Gaia Collaboration 2020, VizieR Online Data Catalog: I/350

Gaustad, J. E., McCullough, P. R., Rosing, W., \& Van Buren, D. 2001, PASP, 113,1326

González-Solares, E. A., Walton, N. A., Greimel, R., et al. 2008, MNRAS, 388, 89

Groot, P. J., Verbeek, K., Greimel, R., et al. 2009, MNRAS, 399, 323

Gvaramadze, V. V. 2006, A\&A, 454, 239

Haffner, L. M., Reynolds, R. J., Tufte, S. L., et al. 2003, ApJS, 149, 405

Haro, G. 1952, ApJ, 115, 572

Herbig, G. H. 1950, ApJ, 111, 11

Høg, E., Fabricius, C., Makarov, V. V., et al. 2000, A\&A, 355, L27

Irwin, M., \& Lewis, J. 2001, New Astron. Rev., 45, 105

Lund, J. M., \& Dixon, R. S. 1973, PASP, 85, 230

Mampaso, A., Corradi, R. L. M., Viironen, K., et al. 2006, A\&A, 458, 203

Miszalski, B., Parker, Q. A., Acker, A., et al. 2008, MNRAS, 384, 525

Monguió, M., Greimel, R., Drew, J. E., et al. 2020, A\&A, 638, A18

Morgan, D. H., Tritton, S. B., Savage, A., Hartley, M., \& Cannon, R. D. 1992, in Digitised Optical Sky Surveys, eds. H. T. MacGillivray, \& E. B. Thomson (Dordrecht: Kluwer Academic Publishers), Astrophys. Space Sci. Lib., 174, 11

Papamastorakis, J., Xilouris, K. M., \& Paleologou, E. V. 1993, A\&A, 279, 536

Parker, Q. A., Phillipps, S., Pierce, M. J., et al. 2005, MNRAS, 362, 689

Parker, Q. A., Acker, A., Frew, D. J., et al. 2006, MNRAS, 373, 79

Parker, Q. A., Bojičić, I. S., \& Frew, D. J. 2016, J. Phys. Conf. Ser., 728, 032008
Sabin, L. 2008, PhD Thesis, School of Physics and Astronomy, Faculty of Engineering and Physical Sciences, The University of Manchester, Manchester, UK

Sabin, L., Zijlstra, A. A., Wareing, C., et al. 2010, PASA, 27, 166

Sabin, L., Parker, Q. A., Contreras, M. E., et al. 2013, MNRAS, 431, 279

Sabin, L., Parker, Q. A., Corradi, R. L. M., et al. 2014, MNRAS, 443, 3388

Sale, S. E., Drew, J. E., Barentsen, G., et al. 2014, MNRAS, 443, 2907

Schlafly, E. F., Green, G. M., Lang, D., et al. 2018, ApJS, 234, 39

Smith, A. R., McDonald, R. J., Hurley, D. C., et al. 2002, in Sensors and Camera Systems for Scientific, Industrial, and Digital Photography Applications III, eds. M. M. Blouke, J. Canosa, \& N. Sampat, SPIE Conf. Ser., 4669, 172

Stecklum, B., Eislöffel, J., Klose, S., et al. 2016, in Ground-based and Airborne Instrumentation for Astronomy VI, eds. C. J. Evans, L. Simard, \& H. Takami, SPIE Conf. Ser., 9908, 99084U

Taylor, M. B. 2006, in Astronomical Data Analysis Software and Systems XV, eds. C. Gabriel, C. Arviset, D. Ponz, \& S. Enrique, ASP Conf. Ser., 351, 666

Taylor, A. R., Leahy, D. A., Tian, W. W., et al. 2017, AJ, 153, 113

Viironen, K., Greimel, R., Corradi, R. L. M., et al. 2009, A\&A, 504, 291

Viironen, K., Mampaso, A., Corradi, R. L. M., et al. 2011, A\&A, 530, A107

Vioque, M., Oudmaijer, R. D., Schreiner, M., et al. 2020, A\&A, 638, A21

Wareing, C. J., O'Brien, T. J., Zijlstra, A. A., et al. 2006, MNRAS, 366, 387

Wesson, R., Barlow, M. J., Corradi, R. L. M., et al. 2008, ApJ, 688, L21

West, R. M. 1974, Eur. South. Obs. ESO Bull., 10, 25

Wolf, C., Onken, C. A., Luvaul, L. C., et al. 2018, PASA, 35, e010

Wouterloot, J. G. A., \& Brand, J. 1989, A\&AS, 80, 149

Wu, C. J., Wu, H., Zhang, W., et al. 2020, Res. Astron. Astrophys., 21, 96 


\section{Appendix A: Image properties and common artefacts}

Here we comment briefly on the known $U_{\mathrm{RGO}}$ and $g$ point spread function (PSF) variations and present an example of a confidence map. We then describe artefacts that one might come across in the survey imagery, especially in lower graded images. These are not unique to the WFC, as they can be found on most imaging instruments. We include this additional material to place it on record for the benefit of future users who may be less familiar with these oddities. The survey data acquisition and pipelining has been described in previous papers (Drew et al. 2005; González-Solares et al. 2008; Groot et al. 2009; Barentsen et al. 2014; Monguió et al. 2020), with relevant aspects summarised here in Sect. 2.

\section{A.1. $U_{\mathrm{RGO}}$ and $g$ PSF variations}

The $U_{\text {RGO }}$ filter stands out among the filters used by the IGAPS survey in being a liquid filter: between $1 \mathrm{~mm}$ UG2 and UBK7 glass plates lies a $5 \mathrm{~mm}$ thick $\mathrm{CuSO}_{4}$ solution.

The liquid nature of the filter leads to different image properties of the $U_{\text {RGO }}$ data. Figure A.1 shows a typical pipeline measured ellipticity distribution for the $g$ filter on the left, as an example representing the four normal glass filters used by IGAPS. The pixel distance to the instrument rotator centre, which should be close to the optical axis, is shown on the $\mathrm{x}$-axis. The data for all 4 WFC CCDs is combined in this plot. A running median is plotted as the thick black line. It can be seen that the ellipticity depends on the radial distance from the optical axis. The right panel of Fig. A.1 shows the same information for the liquid $U_{\mathrm{RGO}}$ filter. A single radial trend does not exist. This leads to a more erratic PSF (see the right-hand panel of Fig. A.2). It is also the reason why an additional fifth-order term is sometimes necessary for the astrometric solution (Monguió et al. 2020). It can also be seen in this example that, despite being a low extinction region, the number of sources visible in the 120 second $U_{\text {RGO }}$ exposure is clearly less than in the 30 second $g$ exposure. The change in point-source image morphology across the WFC array also has an effect on the morphological classification of sources, which are less likely to be classified as stellar the further they are located from the optical axis, independent of the filter (cf. Sect. 2.2 in Farnhill et al. 2016).

As the seal around the edges of the $U_{\mathrm{RGO}}$ filter is not completely tight, the contained solution slowly evaporates over time and hence needs to be topped up whenever an air bubble becomes apparent. Occasionally observations were made with a bubble visible in the filter, which always will drift to the filter edge in zenith direction. At minimum the red leak of the $U_{\mathrm{RGO}}$ filter would be increased for stars observed in the bubble area. Accordingly, bright patches of stars near the edge of frames should be viewed with caution.

During the creation of the IGAPS catalogue it was discovered that there is also an optical blemish on the surface of the $g$ filter, which has an effect on the image quality. The location of this blemish changed over time, depending on the orientation in which the filter was reinserted into the filter holder after cleaning. More information is available in Sect. 6.1 and Appendix B of Monguió et al. (2020).

\section{A.2. Confidence maps}

The confidence maps produced in pipeline processing are used for the masking of bad pixels and vignetted areas on the CCDs.
The pipeline produces the confidence maps per filter from the observations of flat fields taken during an observing run. Hence each IGAPS observing run has its own set of confidence maps associated with it. The confidence map is referenced in the FITS header item CONFMAP (see Appendix B). To define bad pixels, often a limit of confidence $<90$ is used. Figure A.3 shows an example of a confidence map. The vignetting of the image area is clearly visible. The use of a round filter in the WFC has its

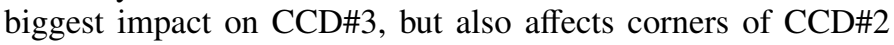
and CCD\#1.

The worst column defects are also visible in the figure. Many thinner column defects and small bad pixel areas are not visible at this image resolution. For specific purposes, such as the selection of diffuse-ISM targets for wide-field spectrographs as described in Sect. 7, the automatic selection of bad pixels via the confidence maps can benefit from the addition of further hot and cold pixels identified by the user.

\section{A.3. Artefacts}

A number of artefacts can be found on the IGAPS WFC images, just as they are in data from a range of astronomical cameras. The cause of these are optical reflections, external particle or light sources, or electronic components.

\section{A.3.1. Bright stars}

For bright stars even the faintest parts of the PSF become visible. The right panel of Fig. 9 shows a bright star and its associated halo, with a radius of $\sim 1100$ pixels. It can be seen that the halo is offset from the star. This offset depends on the distance to the optical axis of the WFC, which hints at the reflection being caused by a curved optical surface. As shown by this example, the halo of a bright star can affect a considerable part of a CCD.

Apart from the direct image and halo, the light of a star also gets reflected onto different optical surfaces of the instrument and forms several large reflections, which can appear more than a degree from the star ('ghosts'). In the case that a star is very bright, these reflections will become visible in the images. One example is shown in the left panel of Fig. 9. The reflection nearly covers a full CCD, and a lot of fine detail from the telescope entrance pupil can be made out - including the cabling of the WFC at the prime focus that protrudes beyond the central obstruction. Also visible is part of a fainter large reflection in the top right corner of the CCD. The seemingly small bipolar nebula at the bottom of that fainter reflection is not real either. That it is just reflected light can be checked by inspecting the offset partner image of the same sky location.

Reflections do not always have to appear complete or have a circular shape. Figure A.4 shows the superpixel map created by HAGRID, as described in Sect. 7.1, as this makes low level detail more readily visible. The left panel of that figure shows two odd reflections. One with a square appearance, which is actually just a cutout from a much larger circular reflection. The other one visible in the top right corner of the CCD is quite different in appearance from the usual circular reflections. The right panel shows part of a circular reflection from a star outside the CCD. On top of it is a smaller, more elliptical reflection. Also visible in this superpixel map is low level fringing outside of the areas covered by the reflections. And the effect of vignetting on CCD\#3 is also clearly discernible.

Figure A.5 shows the effect of a bright star located at or near the edge of a CCD. This leads to the starlight being reflected on to the CCD at an oblique angle. The left panel shows that this 

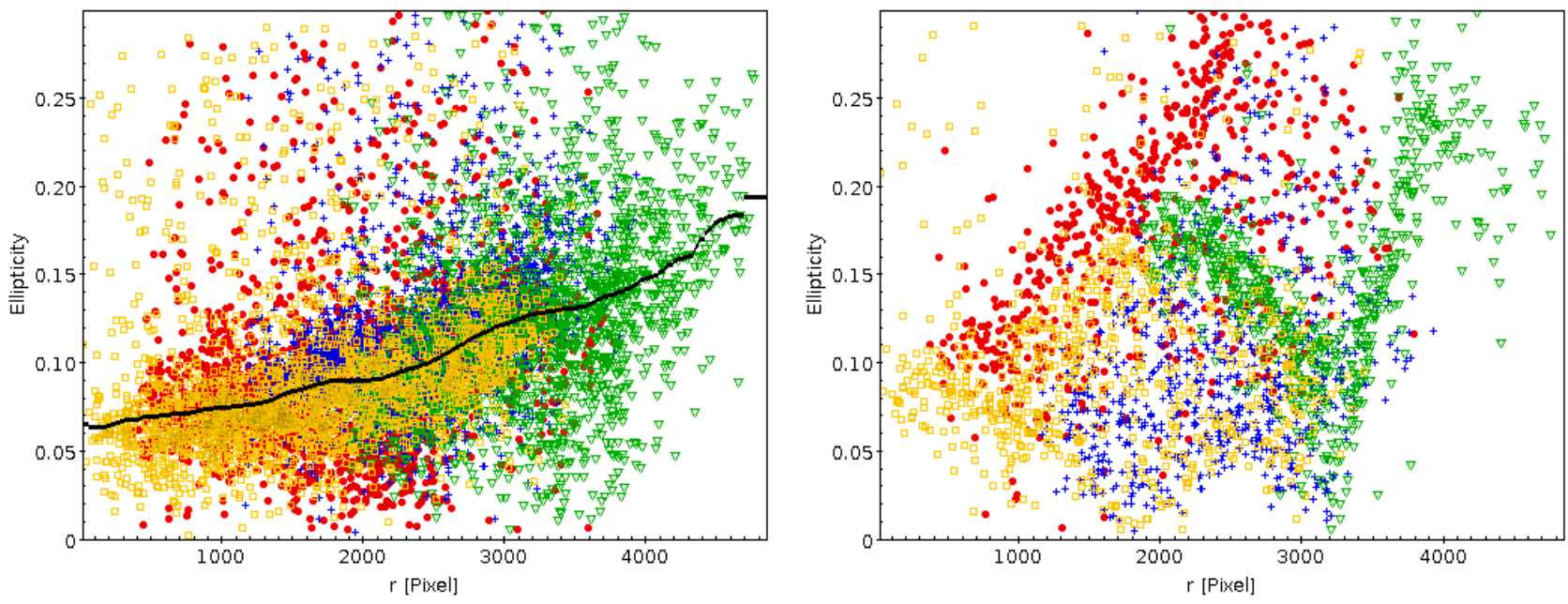

Fig. A.1. Dependence of pipeline measured source ellipticity on the distance to the instrument rotator centre. The data are from IGAPS field 6395o and show the sources from the $g$ image 584231 on the left, and from the $U_{\mathrm{RGO}}$ image r584230 on the right. On the left the data of all four CCDs are shown together with a running median plotted as a black line. On the right the data from each CCD are shown with different colours and symbols: CCD\#1 as red filled dots, CCD\#2 as blue plus signs, CCD\#3 as green triangles, and CCD\#4 as orange squares.

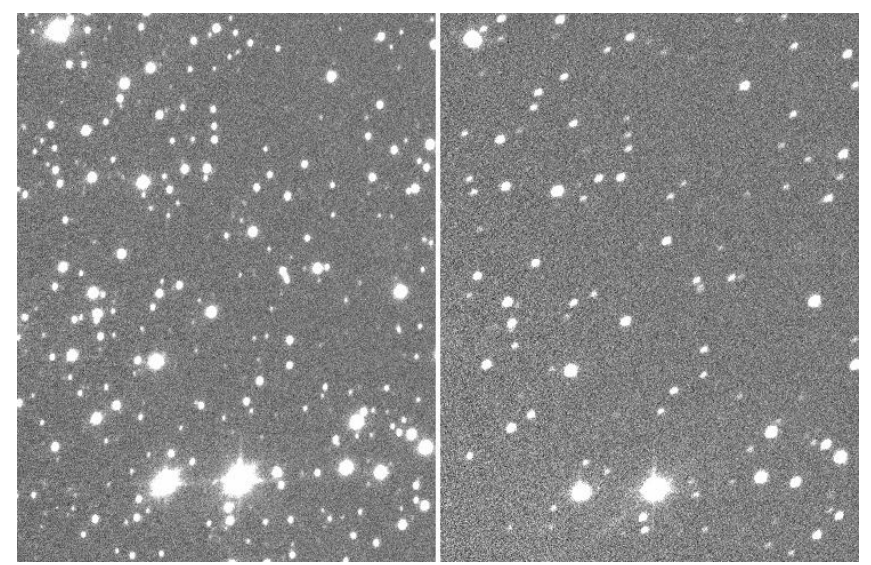

Fig. A.2. Cutout from CCD\#3 of images r584231 ( $g$ filter, left) and r584230 ( $U_{\mathrm{RGO}}$, right), showing the difference in PSF appearance between these filters.

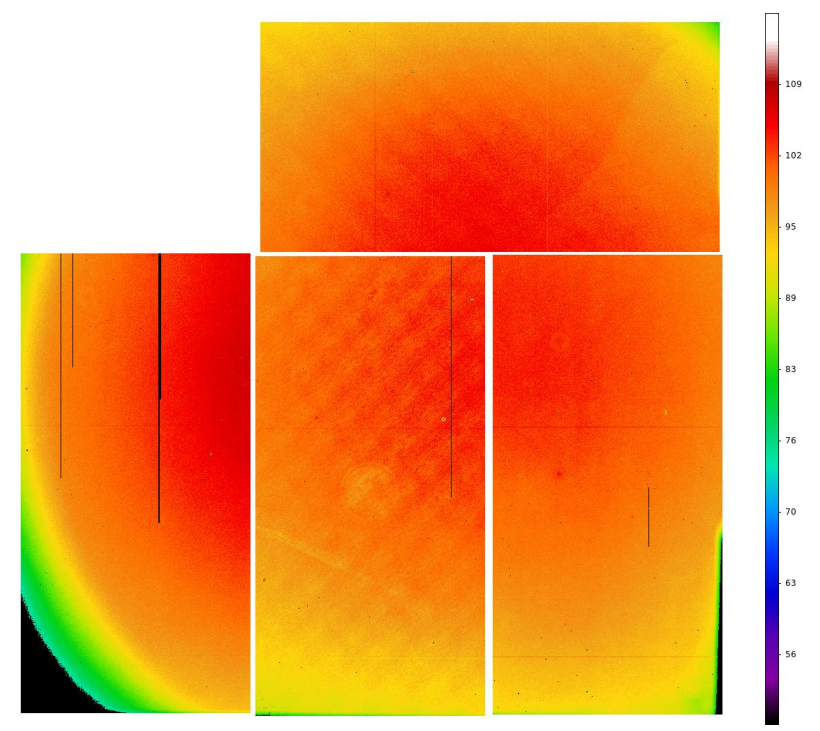

Fig. A.3. Confidence map for the $\mathrm{H} \alpha$ filter from November 2012. Areas shown in black have a confidence value $<50$. The layout of the CCDs has \#2 on top, and \#3, \#4, and \#1 from the left at the bottom.

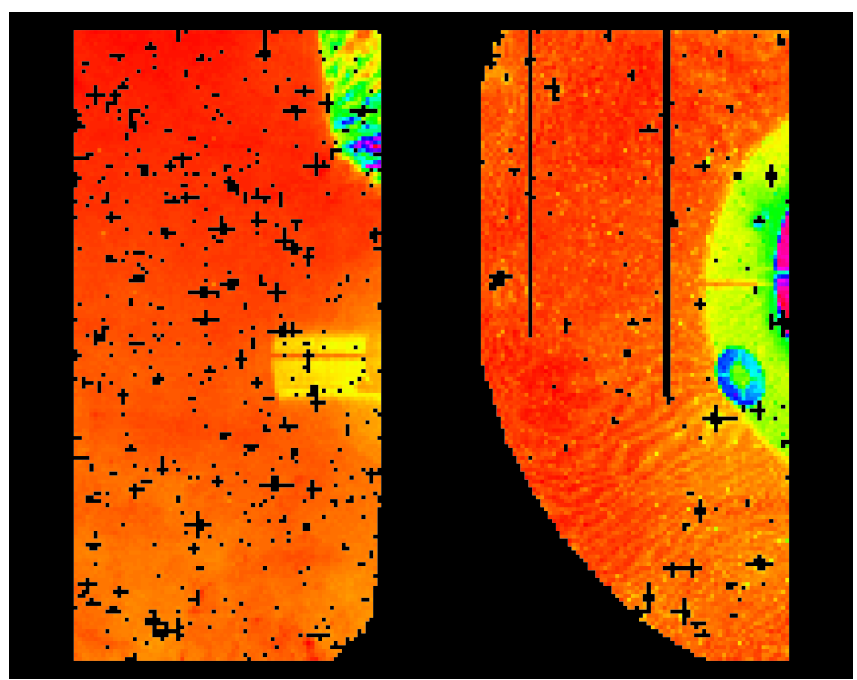

Fig. A.4. Reflections of bright stars outside the CCD. Left: Image $\mathrm{r} 430532, \mathrm{CCD} \# 1, \mathrm{H} \alpha$ filter. The reflection in the centre is up to $25 \%$ of the background. The one near the top reaches more than $300 \%$ of the background counts. Right: Image r764550, CCD\#3, $i$ filter. The large and small reflections have $6 \%$ and $21 \%$ more counts than the background, respectively. The visible fringing is at about $1.5 \%$ of background. The HAGRID superpixel map is shown to enhance low level detail. Black areas correspond to rejected superpixels due to high levels of masking. The colour scale goes from red (low values) to blue (high values).

creates a cometary tail like structure that can extend quite a distance from the star. Also, an almost-square reflection is visible near the top edge, along with a fainter circular reflection. The mask created by HAGRID (cf. Sect. 7.1) is shown in the right panel. It can be seen that this reflection creates a lot of faint spurious sources, picked up erroneously by the IGAPS catalogue, which are arranged in the circular pattern of the reflection. Further spurious sources can be seen extending along the cometary reflection. A further faint part of a circular reflection must exist at the centre of the right CCD edge, as another circular structure of spurious sources can be seen there. 

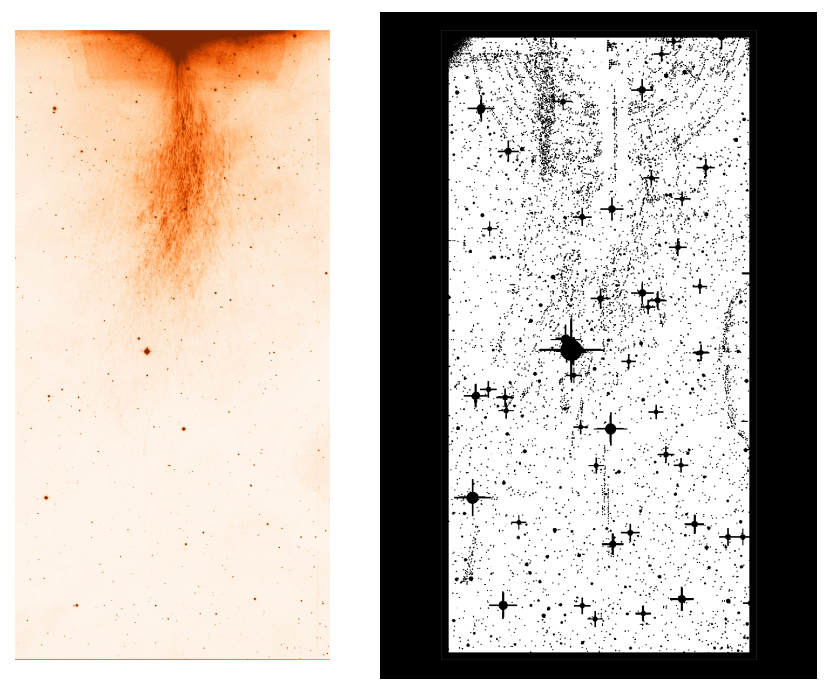

Fig. A.5. Reflection of a bright star on image r414326, CCD\#2. On the left side the $\mathrm{H} \alpha$ image is shown. The pixel mask is shown on the right. Black corresponds to masked pixels. The bright star is located just outside the top edge of the CCD. This creates a cometary-like tail that covers about half the CCD length and a square reflection near the CCD top. The usual circular reflection is also visible at the top. As the masked pixels are generated from the catalogued sources, it can be seen that a lot of faint spurious sources are detected due to the reflections.
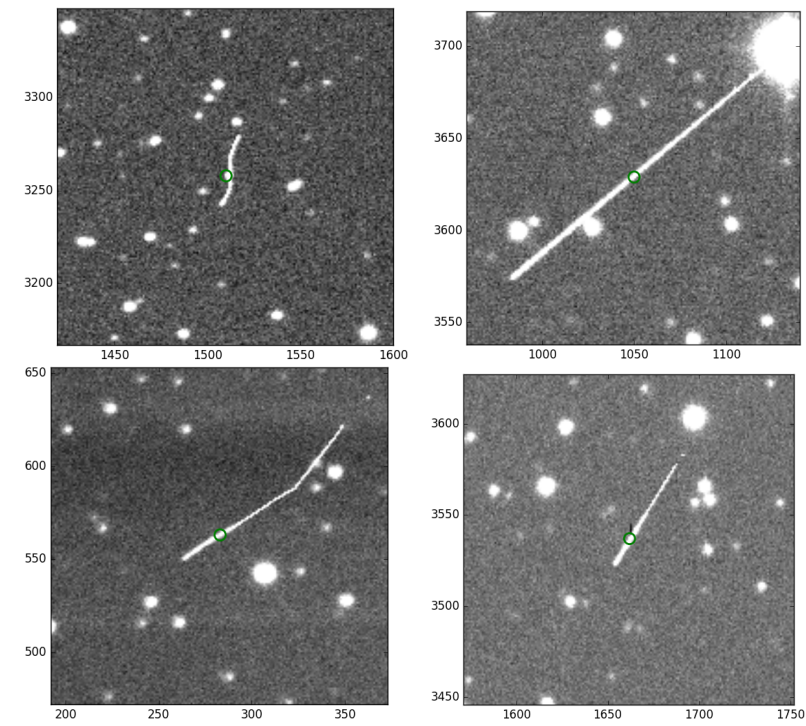

Fig. A.6. Examples of prominent cosmic ray impacts. Top left: Worm caused by multiply scattered low energy electrons. The other three panels show muon impacts. The images are: top left, r372612 CCD\#4 top right, r418359 CCD\#2; bottom left, r431220 CCD\#2; bottom right, r570439 CCD\#3. Each cutout is 180 pixels squared.

\section{A.3.2. Cosmic rays and satellite trails}

Cosmic ray impacts are a well-known nuisance in astronomical images (Smith et al. 2002). Despite their name, not all impacting particles actually are of direct cosmic origin. Figure A.6 shows a few prominent examples of particle impacts visible in the $120 \mathrm{~s}$ $\mathrm{H} \alpha$ exposures. The long streaks visible in the right hand panels are caused by muon impacts. The track in the lower left panel shows a kink in the track, probably due to a collision with a particle in the CCD. The top left panel shows a so called 'worm', caused by multiply scattered low energy electrons.
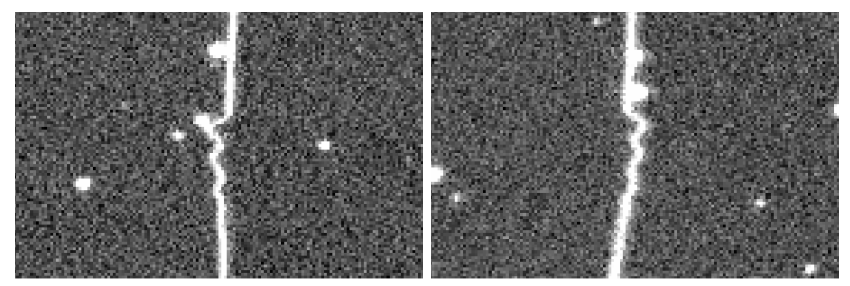

Fig. A.7. Details of the satellite track on the $\mathrm{H} \alpha$ image r541904, CCD\#3. Two excursions away from linear are shown. Telescope tracking or satellite structure might have caused them.

Another nuisance in astronomical images are satellite tracks. With the current and future planned mega satellite constellations in low earth orbit, this problem is very much on the increase. Satellite tracks are mostly straight lines running through the image at an angle. Sometimes flares can be seen, where the brightness increases for a short time due to the alignment of reflecting satellite surfaces with the observing direction. Rather rare is the observation of fine structure in the satellite track. One such example can be seen in Fig. A.7. The cause of these high frequency 'wiggles' may either be due to telescope-tracking glitches in declination or to satellite spin bringing different structures into illumination.

\section{A.3.3. Pickup noise and gain changes}

Occasionally the WFC images suffer from electronic noise, either from external sources (pickup noise) or from readout electronic problems (gain changes). Over time, with ageing electronics, especially the gain changes became more common. The occurrence of gain changes seems to be random, and subsequent images usually are read out correctly. In most cases there is only one gain change during the readout. As certain observing runs had an increased occurrence rate of gain changes, not all of the affected fields could be re-observed at a later date. Hence the data reduction pipeline was modified to deal with the gain changes and still produce a useful object catalogue. An extreme example of many gain changes during the readout is shown in Fig. A.8 in the right panel. These extreme cases could not be salvaged by the data reduction.

The left panel of Fig. A.8 shows an image with typical pickup noise.

\section{A.3.4. Cross talk}

Cross talk during readout is a well-known phenomenon for CCD arrays (Freyhammer et al. 2001). With the WFC, cross talk only becomes visible when a very bright source falls on one of the CCDs. An example is shown in Fig. A.9. The bright star in CCD\#1 creates a negative cross talk in CCD\#2 at a level of about -10 ADU. CCD\#4 shows a positive cross talk at about $10 \mathrm{ADU}$, and CCD\#3 only shows a very small positive cross talk signal in this case.

\section{A.3.5. Multiple images}

Very rarely, multiple images of each source are found on an IGAPS exposure. Normally these appear as double images or streaks. The latter effect is caused by the telescope not being settled on the observing position by the time of exposure start. The former is caused by jumps in the telescope tracking or, in the case of the INT, the oscillation of the main mirror support system (see 

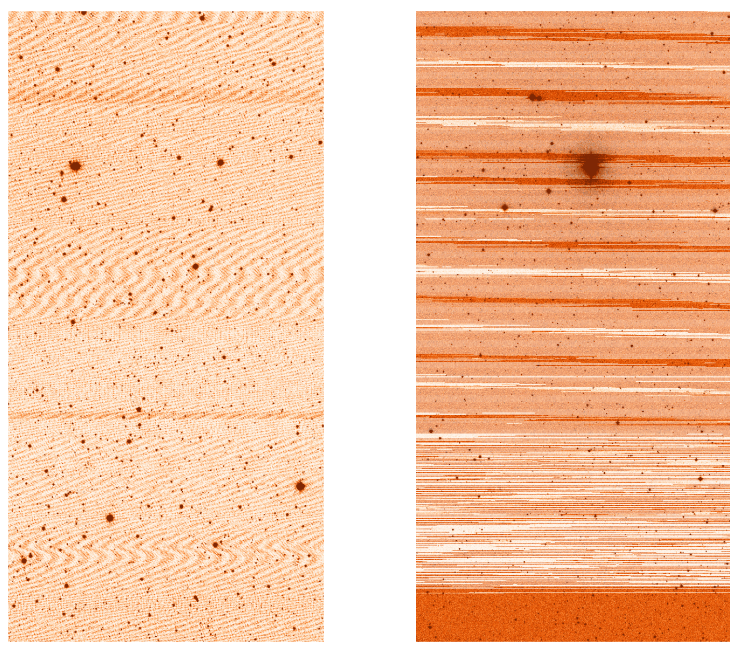

Fig. A.8. Examples of the impact of pickup noise and gain change on CCD images. Left: Pickup noise on image r1018959, CCD\#4. The variations here are small and represent minor degradation. Right: Gain changes on image r1166073, CCD\#4. The changes are both spread across the whole and large, rendering this frame unusable.

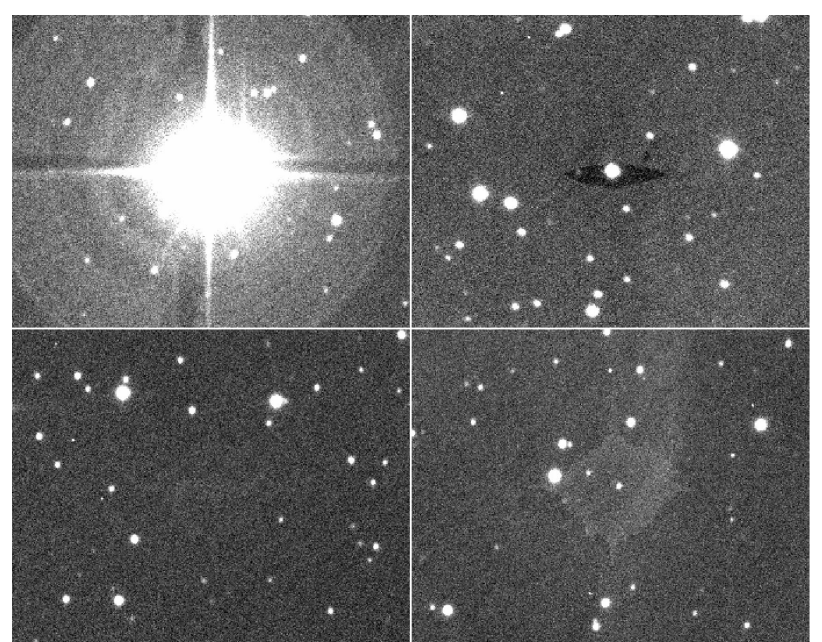

Fig. A.9. Cross talk on image r1023036: CCD\#1 is shown top left, \#2 top right, \#3 bottom left, and \#4 bottom right. The same pixel section is shown for each CCD. The bright star on CCD\#1 is seen as a negative imprint on \#2 for pixels that are saturated, as a positive imprint on \#4, and as a faint positive imprint on \#3.

Fig. A.10). The mirror support system at the INT consists of 36 pneumatic pads, which are controlled together in three $120 \mathrm{deg}$ segments. Oscillations of the servo loop were audible in the control room and could be stopped by the observer by moving the telescope to a different position. It should be noted that due to differences in the time spent at the end points of the oscillation the double images are of different brightness.

\section{A.3.6. Other}

Very occasionally images suffer rare, sometimes unexplained artefacts. Two such examples are shown in Fig. A.11. The left

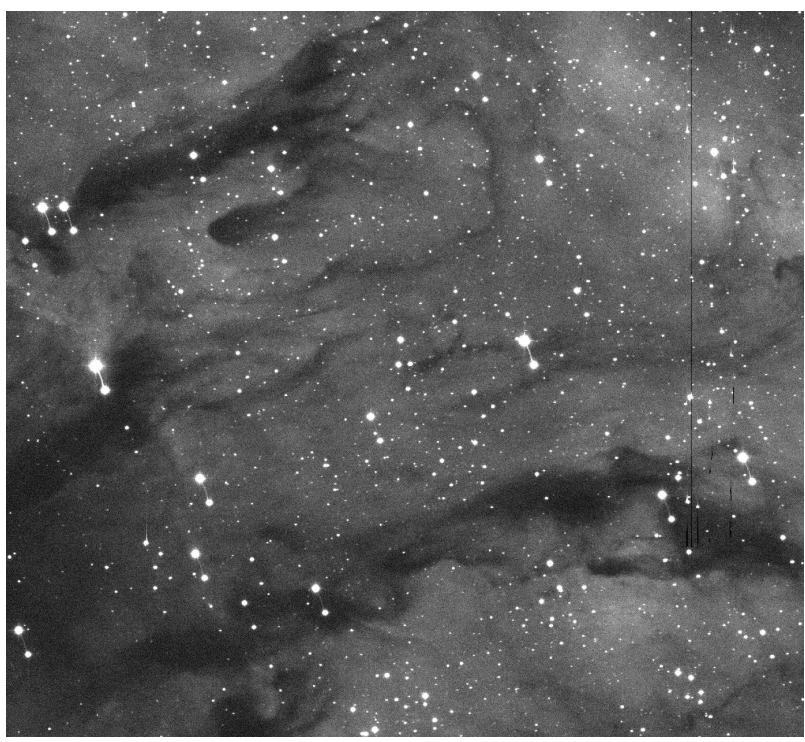

Fig. A.10. Double images on frame r367586, CCD\#4. Bright stars clearly show the sign of INT mirror support oscillation, forming dumbbell-like images.

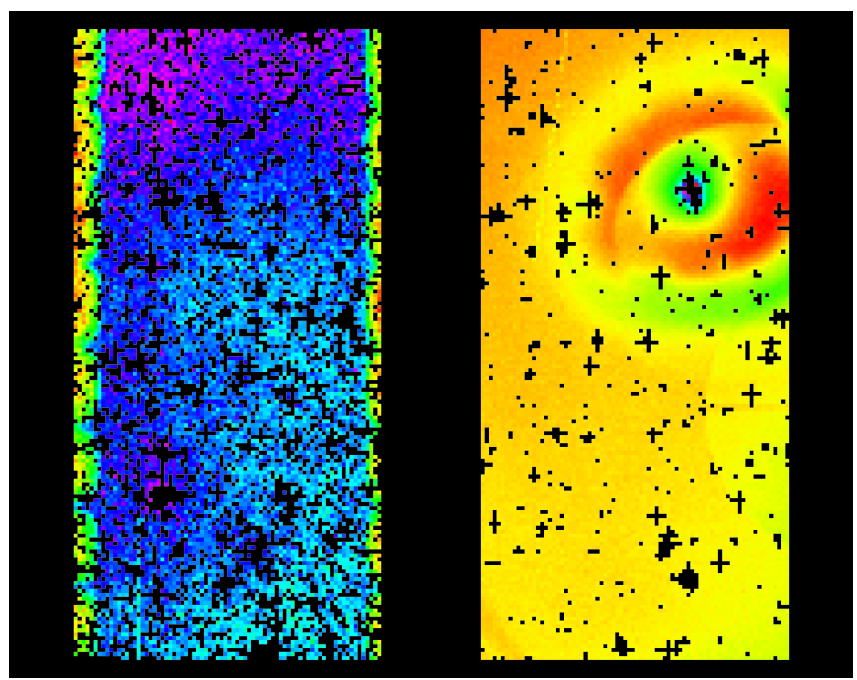

Fig. A.11. Examples of rare image artifacts. Left: Image r418779, CCD\#4. The left and right edges show a 5\% difference in background counts. Right: Image r531376, CCD\#4. The feature, top right, causes up to a $10 \%$ difference relative to the background. The HAGRID superpixel map is shown to enhance low level detail. Black areas correspond to rejected superpixels due to high levels of masking. The colour scale goes from red (low values) to blue (high values).

hand panel shows reduced counts near the left and right edge of the CCD. This effect is visible in CCDs 2 and 4, but not in CCDs 1 and 3 .

The right hand panel of Fig. A.11 shows the effect of a drop of liquid (water or oil) on the filter. This happened during the two nights of 10 and 11 October 2006. The extent and form of the feature changed over time during these nights. 


\section{Appendix B: CCD image header content}

The table below provides an example of the header content associated with each CCD image file. Much of this information is also captured in the metadata table available for download in compressed form as igapsimages.org/data/images/igaps-images.fits.gz.

Table B.1. Image header content.

\begin{tabular}{|c|c|c|}
\hline Parameter & Example value & Explanation \\
\hline BITPIX & 8 & Data type of original image \\
\hline NAXIS & 2 & Dimension of original image \\
\hline NAXIS1 & 2048 & Length of original image axis \\
\hline NAXIS2 & 4096 & Length of original image axis \\
\hline PCOUNT & 0 & Size of special data area \\
\hline GCOUNT & 1 & One data group (required keyword) \\
\hline RUN & 677729 & Run number \\
\hline OBSERVAT & LAPALMA & Name of observatory (IRAF style) \\
\hline OBJECT & intphas_5023 r & Title of observation \\
\hline LATITUDE & 28.761907 & Telescope latitude (degrees) \\
\hline LONGITUD & -17.877559 & Telescope longitude (degrees) \\
\hline HEIGHT & 2348 & [m] Height above sea level. \\
\hline SLATEL & LPO2.5 & Telescope name known to SLALIB \\
\hline TELESCOP & INT & 2.5m Isaac Newton Telescope \\
\hline MJD-OBS & 55015.0452356 & Modified Julian Date at start of observation \\
\hline JD & 2455015.5452356 & Julian Date at start of observation \\
\hline PLATESCA & 6.856013 & {$[\mathrm{~d} / \mathrm{m}]$ Platescale ( 24.68arcsec/mm) } \\
\hline TELFOCUS & 0.043825 & Telescope focus (metres) \\
\hline AIRMASS & 1.021475 & Effective mean airmass \\
\hline TEMPTUBE & 13.508864 & Truss Temperature (degrees Celsius) \\
\hline INSTRUME & WFC & INT wide-field camera is in use. \\
\hline WFFPOS & 5 & Position-number of deployed filter \\
\hline WFFBAND & $\mathrm{r}$ & Waveband of filter \\
\hline WFFID & 214 & Unique identifier of filter \\
\hline SECPPIX & 0.333 & Arcseconds per pixel \\
\hline DETECTOR & WFC & Formal name of camera \\
\hline CCDSPEED & FAST & Readout speed \\
\hline CCDXBIN & 1 & Binning factor in $\mathrm{x}$ axis \\
\hline CCDYBIN & 1 & Binning factor in y axis \\
\hline CCDSUM & 11 & Binning factors (IRAF style) \\
\hline CCDTEMP & 151.959 & {$[\mathrm{~K}]$ Cryostat temperature } \\
\hline NWINDOWS & 0 & Number of readout windows \\
\hline DATE-OBS & 2009-07-03Т01:05:10.8 & Start time of the exposure [UTC] \\
\hline INHERIT & $\mathrm{T}$ & Extension inherits primary HDU. \\
\hline EXTNAME & extension 1 & Extension name \\
\hline EXTVER & 1 & Extension version number \\
\hline IMAGEID & 1 & Image identification \\
\hline DASCHAN & 1 & Number of readout channel \\
\hline WINNO & 0 & Number of readout window \\
\hline CHIPNAME & A5506-4 & Name of detector chip. \\
\hline CCDNAME & A5506-4 & Name of detector chip. \\
\hline CCDCHIP & A5506-4 & Name of detector chip. \\
\hline CCDTYPE & EEV42-80 & Type of detector chip. \\
\hline CCDXPIXE & 0.00001350 & [m] Size of pixels in $x$. \\
\hline CCDYPIXE & 0.00001350 & [m] Size of pixels in $y$. \\
\hline AMPNAME & $\mathrm{LH}$ & Name of output amplifier. \\
\hline GAIN & 2.80000000 & Nominal Photo-electrons per ADU. \\
\hline READNOIS & 6.40000000 & Nominal Readout noise in electrons. \\
\hline SATURATE & 64276.0 & Highest value that is unsaturated \\
\hline BIASSEC & {$[11: 50,3: 4098]$} & Bias pixels. \\
\hline TRIMSEC & [51:2098,3:4098] & Illuminated pixels. \\
\hline
\end{tabular}


Table B.1. continued.

\begin{tabular}{|c|c|c|}
\hline Parameter & Example value & Explanation \\
\hline RTDATSEC & {$[2062: 4215,13: 4212]$} & Location in d-space for RTD. \\
\hline RADESYS & ICRS & WCS calibrated against Gaia-DR2 \\
\hline EQUINOX & 2000.0 & Equinox of the astrometry \\
\hline CTYPE1 & RA ZPN & Algorithm type for axis 1 \\
\hline CTYPE2 & DECZPN & Algorithm type for axis 2 \\
\hline CRUNIT1 & deg & Unit of right ascension coordinates \\
\hline CRUNIT2 & $\operatorname{deg}$ & Unit of declination coordinates \\
\hline PV2_1 & 1.0 & Coefficient for $r$ term \\
\hline PV2_2 & 0.0 & Coefficient for $\mathrm{r}^{* * 2} 2$ term \\
\hline PV2_3 & 213.741679 & Coefficient for $\mathrm{r} * * 3$ term \\
\hline PV2_5 & 0.0 & Coefficient for $\mathrm{r} * * 5$ term \\
\hline CRVAL1 & 292.931917 & [deg] Right ascension at the reference pixel \\
\hline CRVAL2 & 28.6651568 & [deg] Declination at the reference pixel \\
\hline CRPIX1 & -329.738223 & [pixel] Reference pixel along axis 1 \\
\hline CRPIX2 & 2945.36999 & [pixel] Reference pixel along axis 2 \\
\hline CD1_1 & $-1.3972 \mathrm{E}-06$ & Transformation matrix element \\
\hline CD1_2 & $-9.2449 \mathrm{E}-05$ & Transformation matrix element \\
\hline CD2_1 & $-9.2444 \mathrm{E}-05$ & Transformation matrix element \\
\hline CD2_2 & $1.3945 \mathrm{E}-06$ & Transformation matrix element \\
\hline STDCRMS & 0.02526049569007405 & Astrometric fit error $(\operatorname{arcsec})$ \\
\hline MOONDIST & 81.0 & Distance to the moon in degrees \\
\hline MOONALT & 18.29999923706055 & Altitude of the moon above the horizon \\
\hline MOONPHAS & 83.40000152587891 & Phase of the moon \\
\hline SKYLEVEL & 252.99 & Sky level \\
\hline SKYNOISE & 10.96000003814697 & Sky noise \\
\hline PERCORR & -0.005 & Sky calibration correction (mags) \\
\hline MAGZPT & 24.47 & Uncorrected nightly ZP (per second) \\
\hline MAGZRR & 0.02 & Photometric ZP error (mags) \\
\hline EXTINCT & 0.09 & Extinction coefficient (mags) \\
\hline PHOTZP & 28.2187 & $\operatorname{mag}($ Vega $)=-2.5^{*} \log ($ pixel value $)+$ PHOTZP \\
\hline PHOTZPER & 0.03 & Default 1-sigma PHOTZP uncertainty in IGAPS \\
\hline PHOTSYS & Vega & Photometric system \\
\hline FLUXCAL & IGAPS-UNIFORM & Identifies the origin of PHOTZP \\
\hline SEEING & 0.753579 & Average FWHM (arcsec) \\
\hline ELLIPTIC & 0.1319999992847443 & Average ellipticity \\
\hline EXPTIME & 30.07 & [sec] Exposure time adopted \\
\hline CONFMAP & iphas_jul2009 & r_conf.fits Confidence map \\
\hline CHECKSUM & ZfA6ad53VdA3Zd53 & HDU checksum updated 2020-02-11T11:35:56 \\
\hline DATASUM & 1159687462 & data unit checksum updated 2020-02-11T11:35:56 \\
\hline
\end{tabular}

